\title{
Effective parameters for conductive contributions to radial heat transfer in fixed beds under stagnant conditions
}

\author{
Felipe Suárez ${ }^{a}$, Carlos D. Luzi ${ }^{b, c}$, Néstor J. Mariani ${ }^{b, c}$, Guillermo F. \\ Barreto $^{b, c, *}$ \\ a Departamento de Mecánica, Facultad de Ingeniería, UNLP, La Plata, Argentina \\ b PROIRQ Departamento de Ingeniería Química, Facultad de Ingeniería, UNLP, La Plata, Argentina \\ c Centro de Investigación y Desarrollo en Ciencias Aplicadas "Dr. J. J. Ronco" (CINDECA) CCT La Plata - CONICET - \\ UNLP, Calle 47 No. 257, CP B1900AJK, La Plata, Argentina
}

\section{A R T I C L E I N F O}

\section{Article history:}

Received 4 July 2016

Received in revised form 21

December 2016

Accepted 5 January 2017

Available online 16 January 2017

Keywords:

Packed beds

Conductive heat transfer

mechanisms

Heat transfer parameters at

stagnant conditions

Two-Region Two-Dimensional

(2R2D) model

\begin{abstract}
A B S T R A C T
New expressions to evaluate the thermal conduction parameters in fixed beds of monosized spheres are presented in this paper. The parameters are those introduced by the 2R2D model proposed by Asensio et al. (2014): the effective thermal conductivity for the solid core of the bed $\lambda_{\text {es,c }}$ a heat transfer coefficient between the first layer of particles and the wall surface $h_{w p}$ and a heat transfer coefficient between the first layer of particles and inner particles $h_{p L}$. For a better comparison with experimental data those properties are readily associated with the corresponding parameters for stagnant beds $\lambda_{e, c}^{0}, h_{w p}^{0}$ and $h_{p L}^{0}$. Expressions to evaluate the three thermal parameters have been developed on common bases, consisting of a precise approximation for the confluence of flux lines in the particles towards the contact points and packing properties evaluated independently. It is shown that the coefficient $h_{p L}^{0}$ can be directly expressed in terms of $\lambda_{e, c}^{0}$; therefore the practical number of conduction parameters are effectively reduced to $\lambda_{e, c}^{0}$ and $h_{w p}^{0}$. The results from the expressions proposed here for $\lambda_{e, c}^{0}$ and $h_{w p}^{0}$ have been compared with experimental data without employing any fitting parameter. In either case, the analysis of the estimations for particles of nearly spherical shapes did not reveal significant systematic deviations. Experimental information at conditions outside the framework of the theoretical development, such as for rarefied gases and particle shapes other than spherical, was also considered. The analysis of these cases was helpful in assessing effects requiring additional extensions.
\end{abstract}

(c) 2017 Institution of Chemical Engineers. Published by Elsevier B.V. All rights reserved.
1.

Introduction

The correct evaluation of radial heat transport is crucial for simulating the behavior of multi-tubular packed-bed catalytic reactors. Small ratios of tube to particle diameters (aspect ratio, $N=D_{t} / D_{p}$ ), commonly in the range $5<\mathrm{N}<15$, are used in industrial practice to enhance radial heat transfer rates.

Due to the highly nonlinear dependence of catalytic reactions on temperature, and frequently for safety reasons, it has been long ago realized that the mathematical simulation of such units should provide radial and axial temperature distributions in the catalytic packed tubes. The most frequently employed two-dimensional (2D) model assumes radially uniform axial velocity and void fraction, and describes radial heat transfer by means of an also uniform effective thermal conductivity. The model, identified here as Standard Two Dimensional (S2D) model, further introduces a thermal resistance located just at the wall, which includes several effects, such as the thermal resistance due to an unmixed fluid film against the wall, the impairment of radial

\footnotetext{
* Corresponding author.

E-mail address: barreto@quimica.unlp.edu.ar (G.F. Barreto).
} http://dx.doi.org/10.1016/j.cherd.2017.01.012

0263-8762/@ 2017 Institution of Chemical Engineers. Published by Elsevier B.V. All rights reserved. 


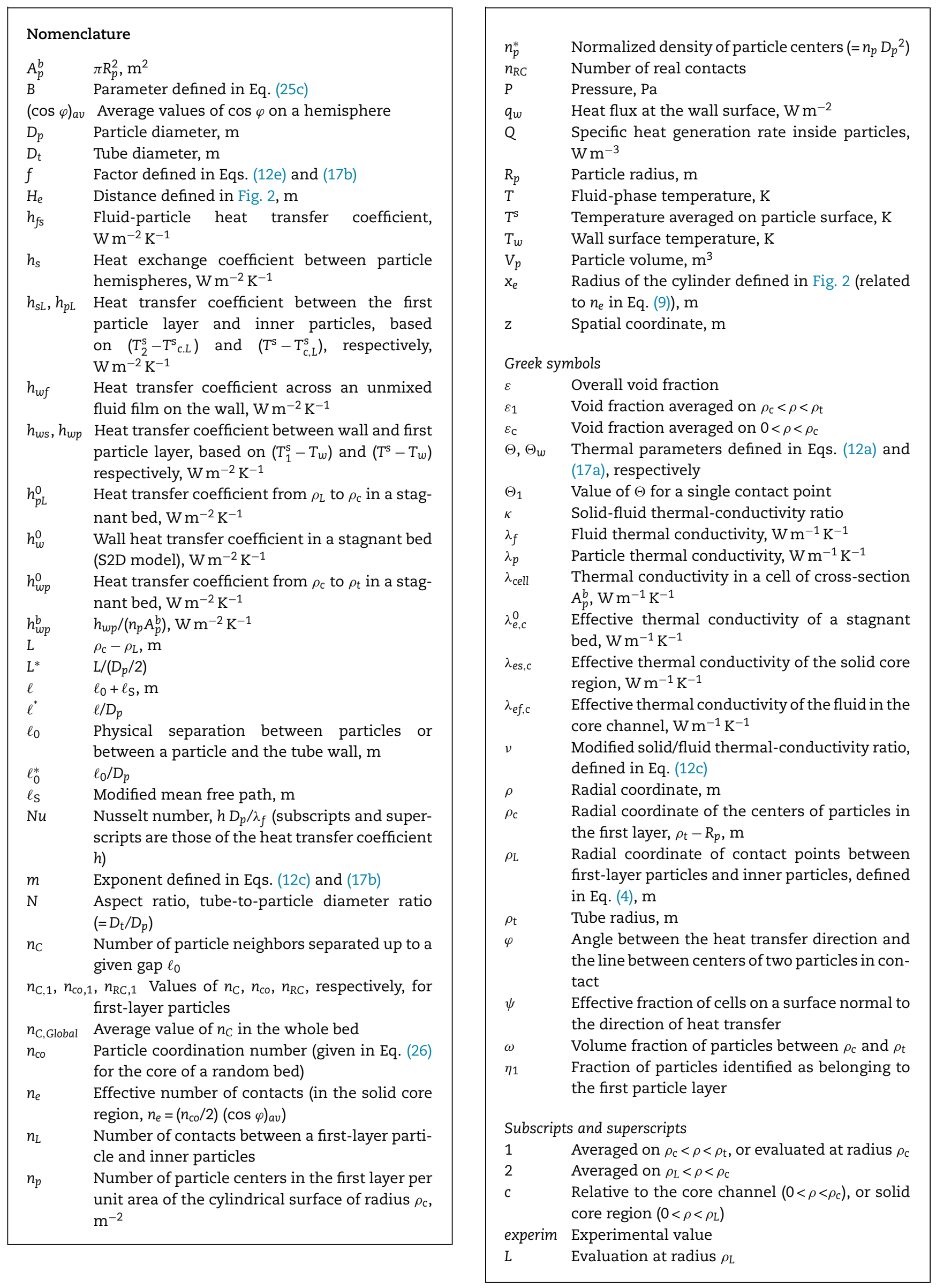




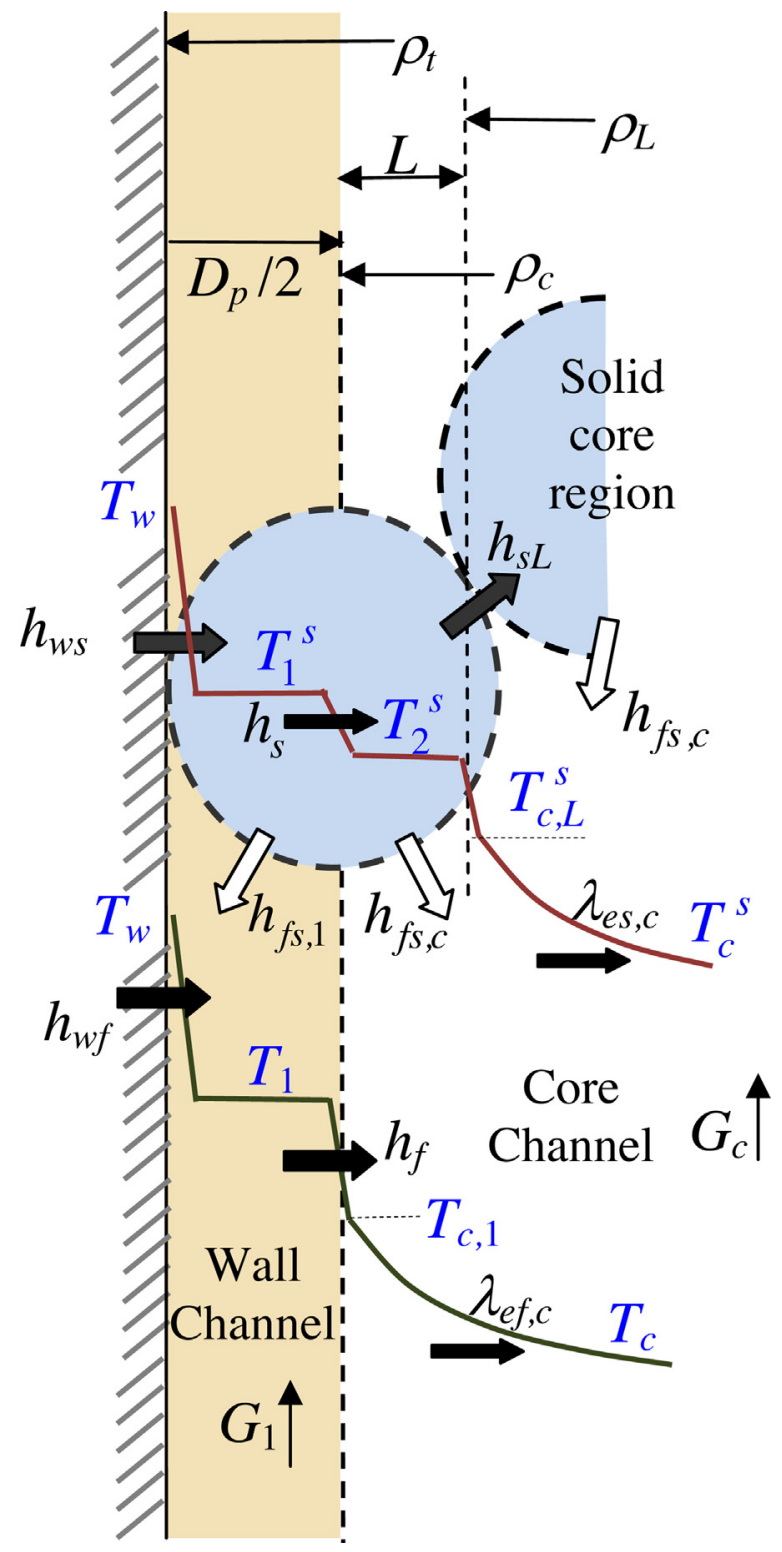

Fig. 1 - Sketch for the 2R2D model of Asensio et al. (2014). The contour of the particle touching the wall represents the first particle layer. The schematic fluid and particle temperature profiles are drawn at different levels.

dispersion of the fluid close to the wall and the existence of a single contact point between the wall and particles touching it (as opposed to several contact points between particles that enhance thermal conductive contributions).

A number of models have been proposed to improve the S2D model by recognizing that the radial heat transfer mechanisms are restrained over a certain distance from the wall (of the order of $D_{p}$ ), instead of considering a concentrated resistance just at the wall (Dixon, 2012; Asensio et al., 2014).

A new model of this type, identified as Two-Region Two-Dimensional (2R2D) model, has been introduced recently by Asensio et al. (2014). The model intends to separate the effects lumped in the concentrated thermal resistance of the S2D model by recognizing in a discrete manner the existence of a well defined layer of particles essentially touching the tube-wall. This feature is very well known for mono-sized spherical particles, and it has also been shown to closely apply in the case of equilateral cylinders (Zhang et al., 2006; Marek, 2013). The existence of such a layer, referred to as "first particle layer", allows identifying a fluid region (wall channel) extended from the wall up to a particle radius (the distance at which the particle centers lie), which presents a significantly higher average voidage $\left(\varepsilon_{1}\right)$ than the rest of the bed $\left(\varepsilon_{c}\right)$ (see Fig. 1).
The high value of $\varepsilon_{1}$ results in a higher permeability in the wall region than in the rest of the bed. Besides, at the boundary between the wall channel and the rest of the bed (i.e., at $D_{p} / 2$ from the wall), a minimum local voidage of around 0.2 severely impairs the lateral exchange of mass between both regions. According to the 2R2D model, the behavior of the inner particles (solid core-region) and the fluid circulating outside the wall channel (core channel) are described in a similar fashion as in the S2D model, i.e. by assuming a pseudo-continuous medium having uniform properties, such as fluid velocity, voidage and effective transport parameters. The concept of a pseudo-continuous medium cannot be sustained for very low values of $N$, for which only a few particles can be accommodated in the inner cross-section of the bed. In this sense, Asensio et al. (2014) suggested tentatively that the use of their model should be limited to $N \geq 5$.

Structural parameters needed for the 2R2D model have been evaluated by Asensio et al. (2014) for monosized spherical particles, taking advantage of many studies that have allowed understanding the structure of packed beds, in particular near the vessel walls. Zambón et al. (2014) have evaluated the main convective parameters of the wall channel by resorting to CFD. In this way, the ratio of superficial mass-velocities in the wall and core channels has been evaluated for Reynolds numbers between values 100 and 2000. The thermal behavior of the wall channel is described by its average temperature. The heat transfer rate at the wall is accounted for by a true film coefficient $h_{w f}$ and heat exchange with the core channel by a coefficient $h_{f}$. Both $h_{w f}$ and $h_{f}$ have been evaluated by Zambón et al. (2014) in the same range of Reynolds numbers and for Prandtl numbers between 0.4 and 3.5 (typical of gases).

This contribution is concerned with the evaluation of the three thermal conduction parameters introduced in the 2R2D model. Two of them quantify the radial heat transfer of the particles in the first layer, with the wall surface $\left(h_{w s}\right)$ and with inner particles $\left(h_{s L}\right)$. The third parameter is the effective thermal conductivity $\lambda_{e s, c}$ describing radial heat transfer between particles in the core region. Two guidelines have been followed to achieve the objective for beds of mono-sized spherical particles and negligible solid deformation at the contact points. The three parameters reflect a common series mechanism consisting in conduction through the particles followed by conduction through nearly motionless fluid fillets interposed between contiguous solid surfaces; therefore, the first guideline is that the prediction of the parameters should be possible by employing the same basic approach. The unique geometrical simplicity of spheres enables to quantify precisely heat conduction through them, and to identify a single geometry around the contacts between spheres or between a sphere and a nearly flat surface. There is at present information available to assess with reasonable precision the packing properties such as the number of contacts of monosized spheres in the core of a bed or close to the wall. The second guideline is to pursue a predictive formulation for the three thermal properties without resorting to adjustable parameters. Maintaining clear physical and geometrical effects in the predictive models is conceptually valuable and we hope it will greatly facilitate extensions to deal with different particle shapes and other modes of heat transfer, such as radiation and conduction through interfaces formed by mechanical contact between solid surfaces. The relationships proposed to estimate $h_{w s}$ and $\lambda_{e s, c}$ in beds of monosized spheres will be validated against experimental data, and $\lambda_{\text {es.c }}$ will be also compared with the very well known expression of Bauer and Schlünder (1978).

Before presenting the steps covering the aims of this contribution, the basic features and expressions of the 2R2D model dealing with conduction contributions will be described in the next section.

It should be mentioned that readers specially interested in the treatment of the effective thermal conductivity may skip over Sections 2, 4 and 5 of this presentation. Besides, it should be made clear that the evaluation of the effective thermal conductivity undertaken in this work is suitable, in addition to the 2R2D model, to any other model resorting to such a concept, and can also be used to account for heat transport in the axial direction of packed beds. 


\section{Highlights of the 2R2D model}

The scheme in Fig. 1 depicts the thermal parameters describing the radial heat transfer mechanisms according to the 2R2D model of Asensio et al. (2014) for a cylindrical packed bed in which catalytic reactions take place inside spherical particles of the same size. The model employs a discrete description for the first particle layer and a pseudo-continuous approach for the rest of the packing (solid core region). Particle surface temperature $T^{s}$ is distinguished from the fluid temperature $T$. For a precise evaluation of the catalytic reaction rates, two particle temperature levels are identified in the first layer: $T_{1}^{s}$ as the average surface temperature over $\rho_{\mathrm{c}}<\rho<\rho_{\mathrm{t}}$, with $\rho_{\mathrm{c}}=\rho_{\mathrm{t}}-D_{\mathrm{p}} / 2$ (Fig. 1), and $\mathrm{T}_{2}^{\mathrm{s}}$ on the remaining part, opposite to the wall. For evaluating the radial distribution of solids, all particles in the first layer are regarded as touching the wall. Then, their centers are separated by a distance $D_{p} / 2=R_{p}$ from the wall. Further relevant features of the $2 \mathrm{R} 2 \mathrm{D}$ models are summarized in the remainder of this section and a more detailed discussion can also be found in Section 3 of Asensio et al. (2014).

The volume fraction of the first-layer particles in $\rho_{\mathrm{c}}<\rho<\rho_{\mathrm{t}}$ is approximately evaluated as

$$
\omega=0.5(1+0.3 / \mathrm{N})
$$

Eq. (1) and further quantification of the packing properties are limited to aspect ratios of $N>5$ (Asensio et al., 2014). The departure of $\omega$ from the value 0.5 is due to the curvature effects. For any practical purpose $\omega$ can be directly taken as 0.5 if $N>10$. The fraction of the external particle surface in $\rho_{c}<\rho<\rho_{\mathrm{t}}$ is also evaluated approximately as $\omega$.

Since inner particles with centers in the interval $\rho_{\mathrm{c}^{-}}$ $R_{p}<\rho<\rho_{c}$ are very scarce (see e.g. Mariani et al. (2002)), only particles in the first layer contribute significantly to the solid fraction $\left(1-\varepsilon_{1}\right)$ in the region $\rho_{\mathrm{c}}<\rho<\rho_{\mathrm{t}}$. Then,

$$
\left(1-\varepsilon_{1}\right)\left(\rho_{\mathrm{t}}^{2}-\rho_{\mathrm{c}}^{2}\right)=\omega n_{p}\left(\frac{\pi}{6} D_{p}^{3}\right) 2 \rho_{\mathrm{c}}
$$

where $n_{p}$ is the number of particles per unit area of the cylindrical surface of radius $\rho_{\text {c }}$. According to Mariani et al. (2002), the dimensionless quantity $n_{p}^{*}=n_{p} D_{p}^{2}$ keeps nearly constant at $n_{p}^{*}=0.955$ in the range $5<N<20$, with deviations not larger than $3 \%$ from measurements in beds of well compacted particles. The same value was also suitable to fit the results from simulated packed beds in the range $5<N<30$ (Salvat et al., 2005).

The void fraction in the rest of the bed $\varepsilon_{c}$ is related to $\varepsilon_{1}$ and the overall voidage $\varepsilon$ as:

$$
\left(1-\varepsilon_{c}\right) \rho_{c}^{2}=(1-\varepsilon) \rho_{t}^{2}-\omega n_{p}\left(\frac{\pi}{6} D_{p}^{3}\right) 2 \rho_{c}
$$

The last structural variable needed for the 2R2D model is the position $\rho_{\mathrm{L}}$ at which the solid core region begins (Fig. 1). As this definition is particularly important for heat exchange between the first particle layer and the core region, it was proposed (Asensio et al., 2014) to relate $\rho_{\mathrm{L}}$ with the radial position of the contact points between the particles in both regions. On this basis, Asensio et al. (2014) established, on one hand, that the volume of first-layer particles remaining at the right of $\rho_{\mathrm{L}}$ should equal the volume of inner particles remaining at the left of $\rho_{\mathrm{L}}$ (see Fig. 1) and, on the other hand, that the voidage in the zone $\rho_{\mathrm{c}}>\rho>\rho_{\mathrm{L}}$ should be $\varepsilon_{\mathrm{c}}$. In this way, the following expression arises for estimating $\rho_{\mathrm{L}}$ :

$$
\left(1-\varepsilon_{c}\right)\left(\rho_{\mathrm{c}}^{2}-\rho_{\mathrm{L}}^{2}\right)=(1-\omega) n_{p}\left(\frac{\pi}{6} D_{p}^{3}\right) 2 \rho_{\mathrm{c}}
$$

Also, for beds of well compacted particles and $N>5$ Asensio et al. (2014) suggested for $\varepsilon_{c}$

$$
\varepsilon_{C}=0.375+\frac{0.13}{N}
$$

Given $n_{p}^{*}$ and $\varepsilon_{c}$, Eqs. (1)-(4) allow calculating all structural properties needed by the 2R2D model. In particular, the overall voidage $\varepsilon$ arises from Eq. (3). It is noted that using $n_{p}^{*}=0.955$ and Eq. (5a), the resulting values of $\varepsilon$ become very close to those from the empirical expression given by Mariani et al. (2002),

$$
\varepsilon=0.375+\frac{0.355}{N}
$$

The energy conservation equations for the first particle layer and for the pseudo-continuous region (Asensio et al., 2014) are:

- In the first particle layer:

$$
\begin{aligned}
& \left(1-\varepsilon_{1}\right)\left(\rho_{\mathrm{t}}^{2}-\rho_{\mathrm{c}}^{2}\right)\left[\mathrm{Q}_{1}-\frac{6 \mathrm{~h}_{\mathrm{fs}, 1}}{D_{p}}\left(\mathrm{~T}_{1}^{\mathrm{s}}-\mathrm{T}_{1}\right)\right]=2 \rho_{\mathrm{t}} \mathrm{h}_{w s}\left(\mathrm{~T}_{1}^{\mathrm{s}}-\mathrm{T}_{w}\right) \\
& \quad+2 \rho_{\mathrm{c}} \mathrm{h}_{\mathrm{s}}\left(\mathrm{T}_{1}^{s}-\mathrm{T}_{2}^{s}\right)
\end{aligned}
$$

$$
\begin{aligned}
& \left(1-\varepsilon_{c}\right)\left(\rho_{c}^{2}-\rho_{L}^{2}\right)\left[Q_{2}-\frac{6 h_{f s, c}}{D_{p}}\left(T_{2}^{s}-T_{2}\right)\right]=2 \rho_{L} h_{s L}\left(T_{2}^{s}-T_{c, L}^{s}\right) \\
& \quad+2 \rho_{c} h_{s}\left(T_{2}^{s}-T_{1}^{s}\right)
\end{aligned}
$$

- In the solid core region $\left(\rho<\rho_{\mathrm{L}}\right)$ :

$$
\begin{gathered}
-\lambda_{e s, c} \frac{\partial\left(\rho \partial T_{c}^{s} / \partial \rho\right)}{\rho \partial \rho}=\left(1-\varepsilon_{c}\right)\left[\frac{6 h_{f s, c}}{D_{p}}\left(T_{c}-T_{c}^{s}\right)+Q_{c}\right] \\
-\lambda_{e s, c} \frac{\partial T_{c}^{s}}{\partial \rho}=h_{s L}\left(T_{c, L}^{s}-T_{2}^{s}\right), \quad \text { at } \quad \rho=\rho_{L} ;
\end{gathered}
$$

$$
\frac{\partial T_{c}^{s}}{\partial \rho}=0, \quad \text { at } \quad \rho=0
$$

In Eqs. (6a), (6b) $Q_{1}$ and $Q_{2}$ are the specific rates of heat released by the catalytic reactions, depending on $\mathrm{T}_{1}^{\mathrm{s}}$ and $\mathrm{T}_{2}^{\mathrm{s}}$ and species concentrations in the regions $\rho_{\mathrm{c}}<\rho<\rho_{\mathrm{t}}$ and $\rho_{\mathrm{L}}<\rho<\rho_{\mathrm{C}}$, respectively (Asensio et al., 2014), while $T_{1}$ and $T_{2}$ are average fluid temperatures in those regions. The role of $Q_{c}$ and $T^{s}{ }_{c}$ in Eq. (6c) for the pseudo-homogeneous region is similar, but on a local basis. The heat transfer coefficients in Eqs. (6a)-(6d) are defined on the basis of the surfaces detailed in Table 1 (see also Fig. 1). 
Table 1 - Surfaces on which heat transfer coefficients are defined.

Heat transfer coefficient

$h_{w s}$

$h_{s}$

$h_{\mathrm{sL}}$

$h_{\mathrm{fs}, 1}, h_{\mathrm{fs}, \mathrm{c}}$

Strictly the heat transfer coefficients pertaining to the first particle layer $\left(h_{w s}, h_{s}, h_{s L}, h_{f s, 1}\right)$ on the one hand, and the parameters of the solid core region $\left(\lambda_{e s, c}, h_{f s, c}\right)$, on the other hand, will be correlated and, in turn, each of them will depend on the specific fluid-dynamics and catalytic reactions taking place. Under such a scenario, the practical usefulness of an up-scaled model such as the 2R2D model will be lost, since the information required for evaluating the effective parameters can only be obtained by means of refined calculations, e.g. from using discrete CFD techniques, for each set of operating conditions. Instead, any up-scaled model should rely upon the evaluation of its parameters under specific conditions able to highlight their significance within the model's frame. In this context, the evaluation of those parameters that quantify the mechanism of conduction from particle to particle $\left(h_{S L}\right.$ and $\left.\lambda_{e s, c}\right)$ and particle to wall surface $\left(h_{w s}\right)$ will be carried out by considering stagnant beds and that the effect of heat generation rate upon such parameters can be neglected.

Asensio et al. (2014) provide further discussion on the 2R2D model. In particular, the use of surface-averaged temperatures (i.e. $T_{1}^{s}, T_{2}^{S}, T_{c}^{s}$ in Eqs. (6a)-(6e)) rather than volume-averaged temperatures are justified in relation to a better quantification of heat exchange between particles and the fluid-stream, and temperature rise/drop inside particles due to reaction heat effects. Also, the use of two-temperature levels in the particles of the first layer imposed the need to include the intra-particle heat transfer coefficient $h_{s}$, which is evaluated according to Asensio et al. (2014) as

$$
h_{\mathrm{s}}=\frac{\pi}{4} n_{p}^{*} \lambda_{p} /\left(D_{p} / 2\right)
$$

\section{Basic approach for evaluating the conductive parameters}

Following the concepts discussed in the former section, to quantify $h_{w s}, h_{s L}$ and $\lambda_{e s, c}$, heat generation will be neglected, the fluid will be assumed to be stagnant and, in the case of a gas, the pressure $P$ will be considered high enough for conduction to be governed by the continuum equations and gas thermal conductivity. The case of rarified gases, however, is considered in Section S4 of the Supplementary material.

The approach outlined below emphasizes cases of relatively high conductivity ratio $\kappa=\lambda_{p} / \lambda_{f}$, although the behavior at the limiting value $\kappa=1$ will be satisfied.

Assuming no contact-area between particles (i.e. solid-solid conduction can be neglected) and a low enough temperature to avoid significant radiant heat transfer, when $\kappa \gg 1$ the overall conductive mechanism through the bed is strongly hindered by the fluid conduction through a small fluid-zone around particle contacts or particle-to-wall contacts. In this way, conduction through multiple contacts on the same particle can be considered as being in parallel and more or less independent of each other. This concept allows

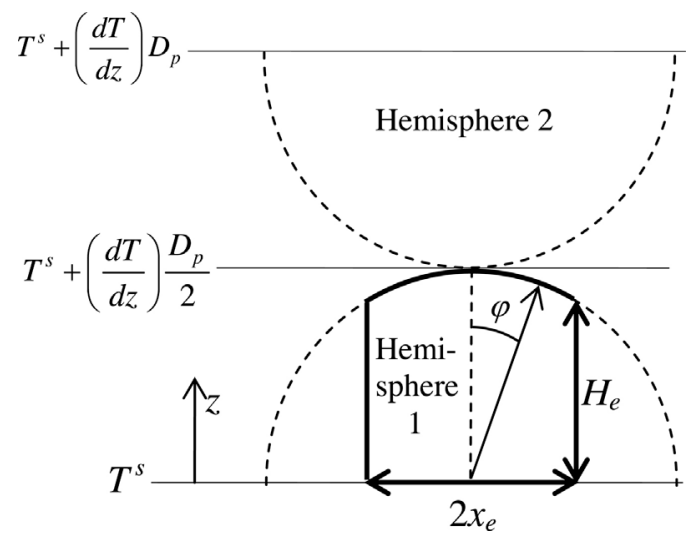

Fig. 2 - Sketch of two contacting hemispheres.

employing the same basic approach for any of the parameters $h_{w s}, h_{s L}$ and $\lambda_{e s, c}$, in spite of the fact that a particle in the core of the bed will present more than one contact, but only a single contact holds between a particle and the wall surface. It should be noted that "contacts" are employed in a broad sense, including real contact points as well as near contacts, i.e. when the surfaces are separated by a small gap. Whenever necessary the distinction will be unambiguously made.

As a matter of convenience, the following treatment focuses on the evaluation of $\lambda_{\text {es }, c}$.

The basic case is posed by a single contact between two hemispheres of the same size with their centers aligned in the direction $z$ of heat transfer, Fig. 2. In evaluating the rate of heat exchanged between the two hemispheres, the fluid volume enclosed by the cylindrical envelope of radius $D_{p} / 2$ is regarded as being adiabatic, as the low value of $\lambda_{f}$ allows ignoring the participation of such a volume in heat transfer between each hemisphere and solid surfaces farther away. This simplification is actually introduced by all unit-cell models, after the classification of Tsotsas and Martin (1987).

For particle 1 in Fig. 2 the reference temperature is the average on its external surface, $T^{S}$. It is shown in Appendix A that for a sphere with uniform and isotropic thermal conductivity, regardless of the type of boundary conditions, $T^{s}$ is exactly equal to the temperature averaged on the volume $V_{p}$, provided that no heat generation inside the sphere takes place. Also, when periodicity applies on the lower and upper hemispheres of each sphere, the temperature at the equator will be uniform and equal to $T^{s}$. Then, the hemispheres 1 and 2 in Fig. 2 are characterized by the equator temperatures $\mathrm{T}^{\mathrm{s}}$ and $T^{s}+(d T / d z) D_{p}$, where $(d T / d z)$ is the imposed temperature gradient. Furthermore, to carry out the thermal analysis, it is only necessary to consider the heat exchanged between the hemisphere 1 and the tangent plane at the contact point, which will present a uniform temperature $T^{s}+(d T / d z) D_{p} / 2$.

The effective conductivity of such a semi-cell will be the same as that between the two hemispheres, $\lambda_{\text {cell }}$, which is defined on the basis of the equator cross-sectional area $A_{p}^{b}=$ $\pi D_{p}^{2} / 4$. If $\lambda_{\text {cell }}$ is evaluated, the result can be expressed as $\lambda_{\text {cell }} / \lambda_{f}=\Theta_{1}$, and it should be verified that $\Theta_{1}=1$ when $\kappa=1$. Now, we should consider the actual case with particles having a total number $n_{c o}$ of contacts. Statistically, hemisphere 1 will present $n_{\mathrm{co}} / 2$ contacts with other hemispheres, each one aligned under a certain angle $\varphi<\pi / 2$ with respect to coordinate $z$ (Fig. 2). Following the notion of independent thermal resistances, all contacts are expected to present the same thermal resistance $\left(\lambda_{f} \Theta_{1}\right)^{-1}$, but the individual thermal driving force will be $\left[\cos (\varphi)(d T / d z)\left(D_{p} / 2\right)\right]$. By adding all contributions, the 
result $\lambda_{\text {cell }} / \lambda_{f}=\left(n_{\text {co }} / 2\right)(\cos \varphi)_{\text {av }} \Theta_{1}$ will be obtained, where (cos $\varphi)_{a v}$ is the average value for $\cos (\varphi)$. Therefore, defining

$$
n_{e}=\left(n_{\mathrm{co}} / 2\right)(\cos \varphi)_{a v},
$$

it can be written $\lambda_{\text {cell }} / \lambda_{f}=n_{e} \Theta_{1}$. This result is expected to hold when $\kappa \gg 1$. However, such an expression for $\lambda_{\text {cell }}$ will be clearly inconsistent when $\kappa \rightarrow 1$, as $\Theta_{1} \rightarrow 1$. To avoid this inconsistency, the approach followed by e.g. Kunii and Smith (1960) is adopted. In this way, the semi-cell considered for evaluating the heat exchange rate is re-defined by the intersection of the hemisphere 1 and the cylindrical surface of radius $x_{e}$ (Fig. 2) such that

$$
\left[x_{e} /\left(D_{p} / 2\right)\right]^{2}=1 / n_{e}
$$

The surface of such a cylinder is again regarded as being adiabatic. In this general case, it will be expressed by adding the contribution of all contacts

$$
\lambda_{\text {cell }} / \lambda_{f}=n_{e} \Theta,
$$

where $\Theta \rightarrow \Theta_{1}$ when $\kappa$ is high enough, and $\Theta \rightarrow 1 / n_{e}$ if $\kappa \rightarrow 1$.

The factor $\Theta$ has been evaluated from the numerical solution of the Laplace equation $\nabla^{2} \mathrm{~T}=0$ using Comsol Multiphysics. To this end, uniform values, $T^{s}$ and $T^{s}+(d T / d z) D_{p} / 2$, on the equator of the hemisphere and on the tangent plane, respectively, were employed. Continuity of temperature and flux in the solid and fluid phases on both sides of the hemispherical surface were imposed. Also, allowance was made for a separation gap $\ell\left(\ll D_{p}\right)$ between the hemispheres, i.e. a distance $\ell / 2$ between the hemisphere 1 and the tangent plane (Fig. 2). This separation accounts for two effects. On the one hand, a possible physical gap $\ell_{0}$ between the two hemispheres (further discussed in the following sections), and, on the other hand, $\ell$ will include the Smoluchowski effect for a gas, which accounts for the fact that closely around a contact the distances between solid surfaces are of the order of the mean free path of the molecules in a gas phase. This effect can be quantified by an equivalent separation $\ell_{S}$, known as modified mean free path. In this contribution the evaluation of $\ell_{S}$ has been made according to Tsotsas and Martin (1987) (the expression is also included in Section 4 of the Supplementary material, Eq. S17). When the fluid is a liquid, $\ell_{S}=0$. Finally, $\ell$ can be expressed as

$$
\ell=\ell_{\mathrm{S}}+\ell_{0}
$$

As no analytical solution is available for the problem just stated, the numerical results have been approximated, in terms of $\Theta$, according to the following explicit formulation,

$$
\Theta=2\left(\frac{v}{v-1}\right)\left[\left(\frac{v}{v-1}\right)\left(1+f \ell^{*}\right) \ln \left(\frac{v\left(f \ell^{*}+1-H_{e}^{*}\right)+H_{e}^{*}}{v f \ell^{*}+1}\right)+H_{e}^{*}-1\right]
$$

where

$$
H_{e}^{*}=H_{e} /\left(D_{p} / 2\right)=\left(1-1 / n_{e}\right)^{1 / 2}
$$

$$
\nu=\kappa^{m} ; \quad m=1+\frac{\ln \kappa}{11.5+1.5 n_{e}}
$$

$$
\ell^{*}=\left(\ell_{\mathrm{S}}+\ell_{0}\right) / D_{p}
$$

$$
f=0.73 n_{e}^{1 / 4}+\left(1-0.73 n_{e}^{1 / 4}\right) / \kappa^{1 / 4}
$$

In Eq. (12b) $H_{e}$ is the distance shown in Fig. 2. Eq. (12a) has been obtained from the analytical solution of the problem assuming flux-lines parallel to the $z$ direction, but with an "effective" solid conductivity given by $\left(\nu \lambda_{f}\right)$ and an "effective" gap given by $(f \ell)$. The parameter $v$ (Eq. (12c)) is introduced to account for the actual curved flux lines in the solid, while the factor $f$ (Eq. (12e)) is similarly related to flux lines in the fluid. The accuracy of Formulation (12a)-(12e) will be discussed in Sections 4 and 6.2 , in relation to the evaluation of $h_{w s}$ and $\lambda_{e s, c}$ respectively.

\section{Evaluation of particle-wall surface heat transfer coefficient}

Neglecting in the first-layer Eqs. (6a), (6b) the terms of heat generation due to catalytic reactions and heat exchange with the fluid, they become

$$
\begin{gathered}
f=0.73 n_{e}^{1 / 4}+\left(1-0.73 n_{e}^{1 / 4}\right) / \kappa^{1 / 4} \\
0=2 \rho_{L} h_{S L}\left(T_{2}^{S}-T_{c, L}^{S}\right)+2 \rho_{c} h_{s}\left(T_{2}^{S}-T_{1}^{S}\right)
\end{gathered}
$$

The flux at the wall surface is $q_{w}=h_{w s}\left(T_{1}^{s}-T_{w}\right)$.

Alternatively, a simpler description based on the average temperature over the whole spherical surface $T^{s}$ can be employed

$$
0=2 \rho_{t} h_{w p}\left(T^{s}-T_{w}\right)+2 \rho_{L} h_{p L}\left(T^{s}-T_{c, L}^{s}\right)
$$

The two terms in Eq. (13c) quantify the heat exchange rates with the wall $\left(\rho=\rho_{\mathrm{t}}\right)$ and with the tangent plane at $\rho=\rho_{\mathrm{L}}$ (Fig. 1). The flux at the wall surface for this alternative description is $q_{w}=h_{w p}\left(T^{s}-T_{w}\right)$.

To establish the relations between the coefficients $h_{w p}$ and $h_{w s}$, and between $h_{p L}$ and $h_{s L}$, the same average temperature and the same flux at the wall from the two approaches are imposed:

$$
\omega \mathrm{T}_{1}^{s}+(1-\omega) \mathrm{T}_{2}^{\mathrm{s}}=\mathrm{T}^{\mathrm{s}}
$$

$$
h_{w s}\left(T_{1}^{s}-T_{w}\right)=h_{w p}\left(T^{s}-T_{w}\right)
$$

Eqs. (13a)-(13c) and (14a), (14b) can be regarded as a set of five equations with five unknowns, $h_{w p}, h_{p L}, T_{1}^{s}, T_{2}^{s}$ and $T^{s}$, for given values of the remaining variables. The solution yields, in particular, the desired relationships between the thermal parameters:

$$
\frac{1}{h_{w p}}=\frac{1}{h_{w s}}+\frac{(1-\omega) \rho_{\mathrm{t}}}{\rho_{\mathrm{c}} h_{\mathrm{s}}}
$$




$$
\frac{1}{h_{p L}}=\frac{1}{h_{s L}}+\frac{\omega \rho_{L}}{\rho_{c} h_{s}}
$$

It is recalled that Eq. (7) provides the estimation for $h_{s}$.

It should be stressed that both approaches are exactly equivalent only when neglecting heat generation and heat exchange with the fluid.

The results from the analysis of Section 3 will be employed next to evaluate the coefficient $h_{w p}$. Once this task is accomplished, the evaluation of the parameter $h_{w s}$ will follow from Eq. (15a). Since all particle centers in the first-layer are separated from the wall by a distance very close to $D_{p} / 2$ and their local environment can be assumed to be reasonably uniform, the consideration of a test particle will be just needed to represent the overall thermal behavior.

In using the results from Section 3 for monosized spheres, a first issue to be addressed regards the reference temperature $\mathrm{T}^{\mathrm{s}}$ used as the uniform temperature on the equator crosssection. Strictly, a test spherical particle in the first-layer will not show a uniform temperature on the equator cross-section, as a consequence of the fact that heat exchange with the wall takes place through a single contact point while heat exchange with inner particles involves a larger number of contacts. However, a comparison of heat transfer rates using a uniform equator temperature and using $\mathrm{T}^{\mathrm{s}}$, carried out by Mazza et al. (1997), showed that differences are smaller than $1 \%$. Therefore, the results in Section 3 can be used with confidence. First, the case when $\mathrm{N} \rightarrow \infty$ will be undertaken. The heat transfer rate between the wall and the test particle can be expressed as

$$
N \rightarrow \infty: \quad h_{w p}^{b} A_{p}^{b}\left(T^{s}-T_{w}\right)=\left[\lambda_{\text {cell }} /\left(D_{p} / 2\right)\right] A_{p}^{b}\left(T^{s}-T_{w}\right)
$$

where the left hand side defines the heat transfer coefficient $h_{w p}^{b}$, and $\lambda_{\text {cell }}$ is the magnitude defined in Section 3 (see Fig. 2) when hemisphere 1 is that of the test particle facing the wall and the tangent plane is considered to be the wall surface at temperature $T_{w}$. In turn, we express $\lambda_{\text {cell }} / \lambda_{f}=\Theta_{w}$, where $\Theta_{w}$ is the specific value of $\Theta$ evaluated from Formulation (12a)-(12e) with a single contact point $\left(n_{e}=1\right)$. Besides, the effective gap $\ell$ (Eq. (11)) included in Formulation (12a)-(12e) is shared by the two hemispheres, but as in the present case only one hemisphere is involved, a factor $(2 \ell)$ rather than $(\ell)$ arises to keep the meaning of $\ell_{0}$ as the minimum distance between surfaces. Explicitly,

$$
\begin{gathered}
\Theta_{w}=2\left(\frac{v}{v-1}\right)\left\{\left(\frac{v}{v-1}\right)\left(1+f 2 \ell^{*}\right) \ln \left[\frac{v\left(1+f 2 \ell^{*}\right)}{1+v f 2 \ell^{*}}\right]-1\right\} \\
\nu=\kappa^{m} ; m=1+\frac{\ln \kappa}{13} ; f=0.73+(1-0.73) / \kappa^{1 / 4} ; \\
\ell^{*}=\left(\ell_{S}+\ell_{0}\right) / D_{p}
\end{gathered}
$$

Values of $N u_{w p}^{b}=h_{w p}^{b} D_{p} / \lambda_{f}=2 \Theta_{w}$ with $\Theta_{w}$ from Eqs. (17a), (17b) are compared with those evaluated numerically (Section 3 ) in Fig. 3. Values obtained by assuming parallel flux lines (normal to the wall surface) in both solid and fluid phases are also plotted in Fig. 3.

Four different values of $\ell^{*}\left(0,10^{-4}, 10^{-3}, 10^{-2}\right)$ are employed. This range of $\ell^{*}$ is of practical significance, as discussed further below. It can be seen that the approximation (17a) produces accurate results. The maximum deviation found by sweeping values of $\kappa$ is around $4 \%$.

The assumption of parallel flux lines for $\ell^{*}=0$ starts to introduce significant errors at $\kappa=10$, while unacceptably low values arise when $\kappa>100$.

The value $\ell^{*}=10^{-4}$ represents the case of no physical-gap $\left(\ell_{0}=0\right)$ and the Smoluchowski effect for air at atmospheric pressure $\left(\ell_{S}=0.2 \mu \mathrm{m}\right)$ and $D_{p}=2 \mathrm{~mm}$. In this typical case, the assumption regarding the flux lines is also inappropriate, except at very large values of $\kappa$ (say, $\kappa>5000$ ) when the solid presents a very low thermal resistance. Actually, in all cases the main source of errors from the assumption of parallel flux lines is due to the solid phase.

It therefore seems that any reliable tool for predicting conductive heat transfer in fixed beds should somehow include the effect of spreading/constriction of flux lines within the particles. The curvature of flux lines in the fluid (accounted for by the factor $f$ in Eq. (17a)) exerts a much weaker effect, which is nearly negligible when $\ell^{*}=0$, but increases up to around $10 \%$ in the range $10^{-3}<\ell^{*}<10^{-2}$ and at high values of $\kappa$.

Following the concept of a "test particle", knowing $h_{w p}^{b}$ allows evaluating $h_{w p}=h_{w p}^{b}\left(n_{p} A_{p}^{b}\right)$. Yet, at this point our estimation of $h_{w p}^{b}$ is subject to the case $N \rightarrow \infty$. As $h_{w p}$ is defined on the basis of the wall-surface area and the area $\left(n_{p} A_{p}^{b}\right)$ is related to the cylindrical surface enclosing the particle centers, a factor $(\mathrm{N}-1) / \mathrm{N}=\rho_{\mathrm{c}} / \rho_{\mathrm{t}}$ (see Fig. 1) should be introduced to evaluate $h_{w p}$ when $N$ is finite. In addition, it should be considered that due to the curvature of the wall the distance from any point on the particle surface to the wall is shorter than when the wall is plane. A geometrical analysis shows that this effect can be accounted for approximately by introducing a further factor $\mathrm{N} /(\mathrm{N}-0.5)$, which can be applied if $\mathrm{N}$ is not very small (safe, if $N>5$ ). It is noted that this factor is very close to that proposed by Legawiec and Ziółkowski (1994) on the basis of numerical evaluations. Approximating the product of both factors discussed above in the way $(N-1) /(N-0.5) \approx(1-0.5 / N)$ enables us to write when $\mathrm{N}>5$,

$$
N u_{w p}=h_{w p} D_{p} / \lambda_{f}=\frac{\pi}{4} n_{p}^{*} 2 \Theta_{w}(1-0.5 / N)
$$

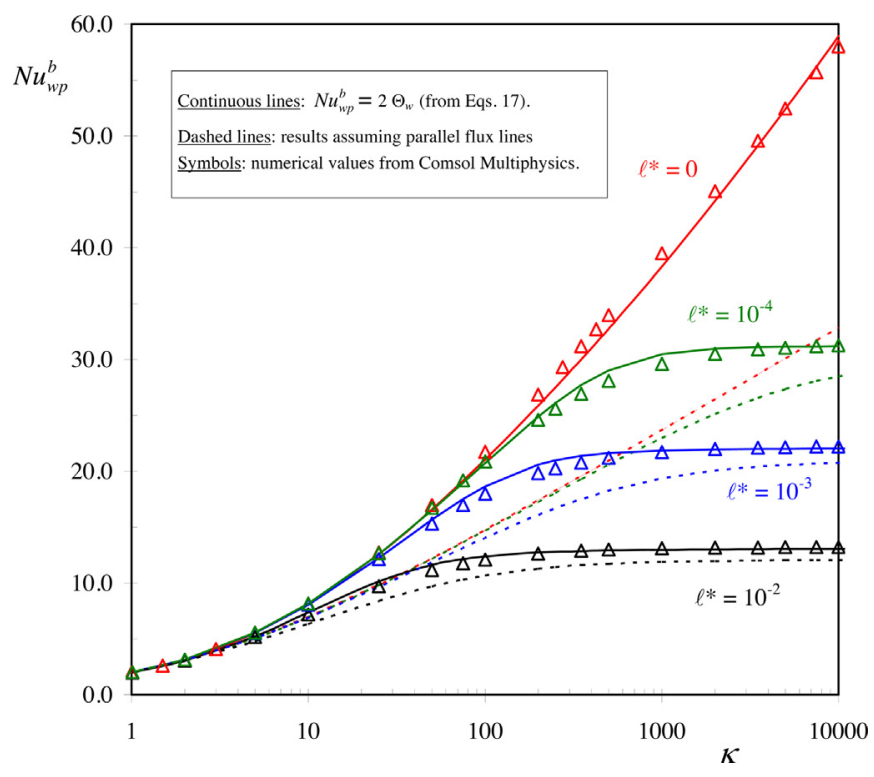

Fig. $3-N u_{u p}^{b}$ vs $\kappa$. 
From Fig. 3 it is evident that even short detachments $\ell_{0}$ of the particles from the wall will cause a significant effect on $\mathrm{Nu}$ wp. Information on this issue is scarce, to the best of our knowledge. Legawiec and Ziółkowski (1994) reported from direct measurements that all particles belonging to the first layer are in effective contact with the wall, but the precision of their instrument was $10 \mu \mathrm{m}$. If $\ell_{0}=10 \mu \mathrm{m}$ is admitted as a possible gap for some particles and assuming $D_{p}=1 \mathrm{~mm}\left(\ell^{*}=0.01\right)$, their contribution to the overall rate of heat transfer will be severely diminished (see Fig. 3). Therefore, no definite conclusion can be drawn in this respect from the work of Legawiec and Ziółkowski (1994). The number of contacts is analysed in Section 6.1 for particles in the core region. It is shown that for a reasonable estimation of the coordination number of a given particle, only around $80 \%$ of the neighbours are in real contact, while the remaining $20 \%$ keep distances of about $0.01 D_{p}$. This imperfect accommodation can also be expected to happen for particles in the first layer. Some indirect evidence can be extracted from experimental estimations of $h_{w p}$. The available information from steady state experiments will be discussed later in this section. On the other hand, transient heat transfer at short contact times is controlled by the first particle layer, and some time ago Mazza et al. (1997) analyzed available experimental information on the basis of a transient model that shares similar effects as accounted for by Eqs. (17a), (17b) and (18). They concluded that separation gaps in the range $0<\ell_{0}<2 \mu \mathrm{m}$ render a good match with most experimental data from different sources. Nonetheless, Mazza et al. (1997) employed a uniform value $\ell_{0}$ for all particles in the first layer. It is physically better to consider that only a fraction of the particles can be slightly detached from the wall. Actually, if $80 \%$ of the particles are assumed in actual contact and $20 \%$ maintain a gap $\ell_{0}=0.01 D_{p}$ (i.e. as for core region particles), the results from Eqs. (17a), (17b) and (18) are very close to those using a uniform value $\ell_{0}=1 \mu \mathrm{m}$, for $D_{p}=2 \mathrm{~mm}$ and values $\kappa<200$. That choice is then compatible with the conclusions of Mazza et al. (1997). Therefore, the expression here proposed to estimate $h_{w p}$ is

$$
N u_{w p}=h_{w p} D_{p} / \lambda_{f}=\frac{\pi}{4} n_{p}^{*} 2\left(0.8 \Theta_{w}^{0}+0.2 \Theta_{w}^{0.01}\right)(1-0.5 / N)
$$

where the superscripts of $\Theta_{w}$ (i.e. 0 and 0.01) stand for the ratios $\left(\ell_{0} / D_{p}\right)$ to be used in (17b).

At steady state conditions and particularly for relatively low values of $\kappa$, the direct fluid conduction from the regions surrounding the first-layer particles cannot be neglected. Assuming that at a distance $R_{p}$ from the wall the fluid takes the same temperature as the average of the first-layer particles (i.e., $\mathrm{T}^{\mathrm{s}}$ ) and that between the wall and those regions the heat is exchanged without any influence by the particles, the following expression is proposed from Eq. (19) to account for the heat transfer rate between the whole surface at $\rho_{c}=\rho_{t}-R_{p}$ and the wall at $\rho_{t}$ :

$$
\begin{aligned}
& N u_{w p}^{0}=h_{w p}^{0} D_{p} / \lambda_{f}=2\left[\left(1-\frac{\pi}{4} n_{p}^{*}\right)+\frac{\pi}{4} n_{p}^{*}\left(0.8 \Theta_{w}^{0}+0.2 \Theta_{w}^{0.01}\right)\right] \\
& (1-0.5 / N)
\end{aligned}
$$

As $\Theta_{w}^{0}, \Theta_{w}^{0.01} \rightarrow 1$ when $\kappa \rightarrow 1$, the stagnant heat transfer coefficient $h_{w p}^{0}$ in Eq. (20) will tend correctly to $h_{w p}^{0}=\lambda_{f} /\left(D_{p} / 2\right)$ $(1-0.5 / \mathrm{N})$, i.e. as for a homogeneous medium with conductivity $\lambda_{f}$.
To summarize the formulation in the present section, it is noted that the relevant expressions to evaluate the related coefficients $h_{w s}, h_{w p}$ and $h_{w p}^{0}$ are Eqs. (15a), (19), (20).

Ofuchi and Kunii (1965) and Melanson and Dixon (1985) carried out steady state experiments at atmospheric pressure of heat transfer through stagnant beds between two surfaces at different temperatures. Flat horizontal surfaces (source and sink) were employed by Ofuchi and Kunii (1965), and they reported experimental values of the product $\left[h_{w p}^{0}\left(D_{p} / 2\right)\right]$. The heat flux $q_{w}$ was measured by a marble plate flux-meter, which also supported the packed beds. Measurements of temperatures at the supporting marble surface therefore provided the experimental values of $T_{w}$, which can be regarded as average values of the wall temperature. Eqs. (18)-(20) are based on the assumption of a uniform wall temperature, a case that frequently can be ensured by metal surfaces with high thermal conductivities. If not, the local wall temperatures close to the contacts points with the particles, where very large local heat transfer coefficients hold on the bed side, can vary significantly from the average temperature $T_{w}$, and become closer to $T^{s}$. Therefore, the local fluxes decrease and an expression like Eq. (20) will overestimate the actual average heat flux evaluated on the basis of a uniform temperature $T_{w}$. The effect, called spreading/constriction thermal resistance, can be expected to be significant in the present case, as the thermal conductivity of marble is relatively low $(2.08 \mathrm{~W} / \mathrm{mK})$. Then, values of $h_{w p}^{0}$ given by Eq. (20) have been corrected according to a procedure recently proposed by Luzi et al. (2015). The Supplementary material (SM) contains details of such a correction (Section S2), which is needed for the Ofuchi and Kunii (1965) data but not for the results of Melanson and Dixon (1985). Also, in Section S3 of the SM the complete set of experimental data from Ofuchi and Kunii, (1965) and Melanson and Dixon (1985) are presented in tabular form, as well as the results predicted using the model, additional comments and fluid properties used to simulate Ofuchi and Kunii (1965) and Melanson and Dixon (1985) experimental results.

The results from Melanson and Dixon (1985) were measured in annular stagnant beds between two concentric surfaces. Wall effects were evaluated at both surfaces, but here we are only interested in the results for the outer surface $\left(\rho_{t}=37.5 \mathrm{~mm}\right)$. Values of the wall heat transfer coefficient $h_{w}^{0}$ corresponding to the standard two-dimensional (S2D) model were reported, but values of $h_{w p}^{0}$ can be retrieved (see Eq. S6 in the SM). The experimental results from Ofuchi and Kunii (1965) are displayed in Fig. $4 \mathrm{a}$, according to the ratio $\kappa$ of the combinations material/fluid. Only one set of results from Melanson and Dixon (1985) is presented, for the sake of clarity in the plot (their remaining results are included in Fig. 4b).

Different experimental values for the same combination of solid material/fluid correspond either to replicates or to different particles sizes. No definite trend is found as regards particle size. Also, the effect of $D_{p}$ on values predicted by Eq. (20) was found to be very mild for the ranges employed in both sources, Ofuchi and Kunii (1965) and Melanson and Dixon (1985); therefore results from Eq. (20) with the average size for each combination of solid material/fluid are used in Fig. 4 a.

It should be stressed that the only bed structural parameter involved in Eq. (20) is $n_{p}^{*}$, for which the value $n_{p}^{*}=0.955$ suggested in Section 2 has been employed. The continuous curve in Fig. $4 \mathrm{a}$ intends to show the expected trend of $N u_{w p}^{0}$ as $\kappa$ increases. It was obtained from Eq. (20) (without spreading/constriction correction) using $\ell_{S} / D_{p}=0.9 \times 10^{-4}$ and $N \rightarrow \infty$ 
a

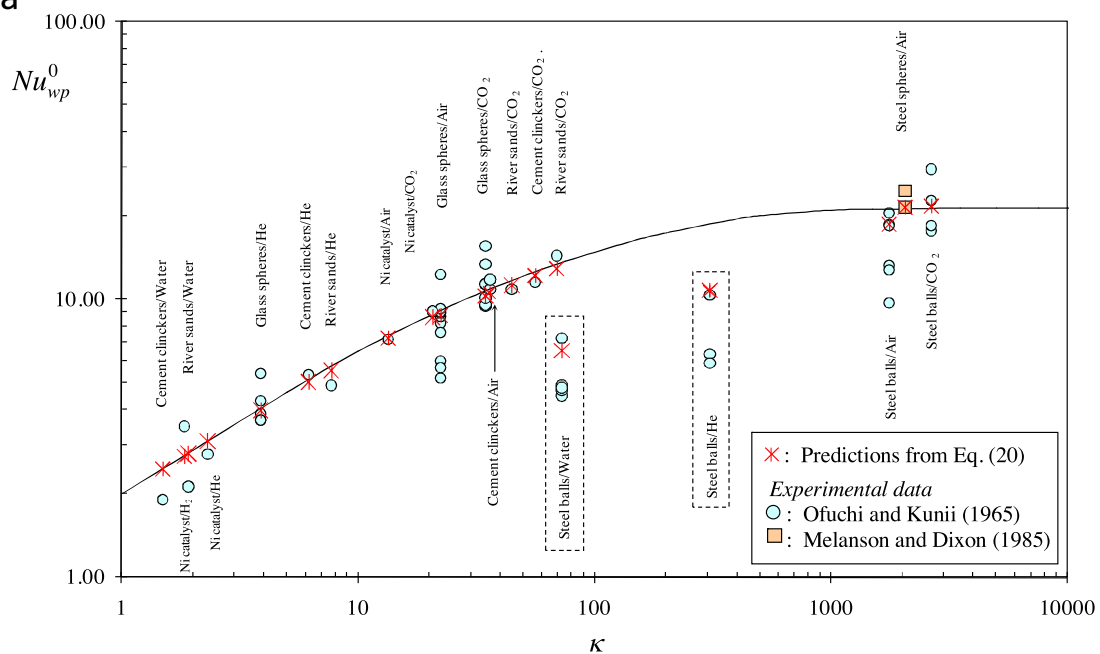

b

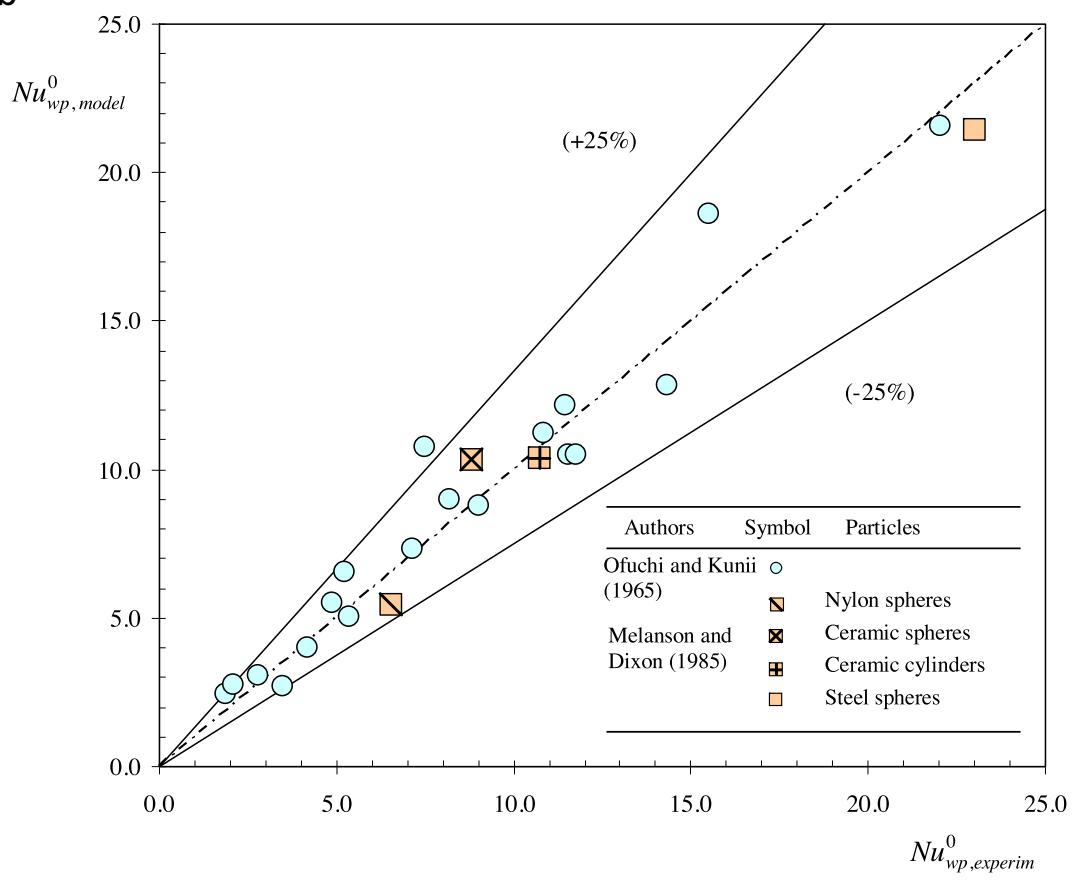

Fig. 4 - (a) $N u_{u p}^{0}$ vs $\kappa$. The continuous curve corresponds to values from Eq. (20) with $\ell_{S} / D_{p}=0.9 \times 10^{-4}, N \rightarrow \infty$ and without spreading/constriction correction. (b) Parity plot. $\mathrm{N} u_{u p, \text { model }}^{0}$ (Eq. (20), corrected values for Ofuchi and Kunii (1965) data) vs. $\mathrm{N} u_{u p \text {, experim. }}^{0}$

for the curvature factor $(1-0.5 / N)$. It can be seen that the spreading/constriction effect turns out to be very important for the combination steel/water and steel/He (the corrected values of $N u_{w p}^{0}$ are $48 \%$ and $68 \%$ lower than those predicted by Eq. 20, respectively), i.e. those with the highest solid and fluid thermal conductivities. The spreading/constriction correction was practically negligible for the rest of the data presented by Ofuchi and Kunii (1965).

The predicted results in Fig. 4 a seem to describe the experimental data obtained by Ofuchi and Kunii (1965) correctly. This impression is reinforced in the parity plot of Fig. $4 \mathrm{~b}$, where the average value for each combination of solid material/fluid has been considered from both sources, Ofuchi and Kunii (1965) and Melanson and Dixon (1985). Defining a relative error $e=100$ $\left(N u_{w p, \text { model }}^{0}-N u_{w p \text {,experim }}^{0}\right) / N u_{w p \text {,experim }}^{0}$ for each of the 23 combinations of solid material/fluid in Fig. $4 \mathrm{~b}$, the arithmetic mean and the average of the absolute values are $e_{a v}=+5.7 \%$ and $|e|_{a v}=13 \%$. The value $e_{a v}=+5.7 \%$ suggests that Eq. (20) overpre- dicts $N u_{w p}^{0}$, but at an acceptably low level. Recalling that Eq. (20) has been employed without tuning any parameter, the low values of $e_{a v}$ and $|e|_{a v}$ provide strong support for the conceptual bases leading to this equation.

It should be noted that although the shapes of cement clinker and river sand particles employed by Ofuchi and Kunii (1965) were not reported, it is most probable that an irregular shape is involved in both cases. It can be seen in Fig. $4 a$ and $b$ that their behavior cannot be distinguished from that of spherical particles. Also included in the present comparison are the sets of data for the cylindrical Ni catalyst (Fig. 4a) from Ofuchi and Kunii (1965) and for a cylindrical ceramic (Fig. 4b) from Melanson and Dixon (1985). It can be appreciated in Fig. $4 \mathrm{a}$ and $b$ that values of $N u_{w p}^{0}$ for both cylindrical particles are in line with those of the spherical particles and Eq. (20) provides a good approximation to them. This result was not foreseen, as many cylinders touch the wall with their axis almost parallel or normal to the wall (see e.g. (Zhang et al., 2006)), leading 
to nearly line or surface contacts that are expected to reduce the effect of the interposed gas gaps and thus to increase the heat transfer rate. Yet, other effects should be appraised, such as values of $n_{p}$ and/or the percentage of real contacts with the wall, which could be different from those for spherical particles. From the analysis of the behavior of all solid and hollowed cylinders tested by Ofuchi and Kunii (1965) and Melanson and Dixon (1985) it appears that when $\kappa$ is relatively low (ceramic particles), the values of $h_{w p}^{0}$ are close to those for equivalent spheres, as discussed above, but when $\kappa$ is high (metallic particles) significantly higher experimental values of $h_{w p}^{0}$ can arise (see Section S3 of the SM for details).

\section{Evaluation of the heat transfer coefficient between the first particle layer and the core region}

We will undertake in this section the evaluation of the coefficient $h_{p L}$, while the related coefficient $h_{s L}$ can be obtained by using Eq. (15b). Asensio et al. (2014) conjectured that heat exchange between the first particle layer and the core region should resemble heat transfer inside the latter, and proposed to estimate $h_{p L}=\lambda_{e s, c} / L$, where $L=\rho_{c}-\rho_{L}$ (see Fig. 1). That expression accounts for the thermal resistance of a slab of thickness L. If the curvature of the bed is considered (i.e. for low values of $\mathrm{N}$ ) a suitable correction is

$$
h_{p L}=\frac{\lambda_{e s, c}}{\rho_{L} \ln \left(\rho_{c} / \rho_{L}\right)}=\frac{2 \lambda_{e s, c}}{D_{p}\left(N-1-L^{*}\right) \ln \left(\frac{N-1}{N-1-L^{*}}\right)}
$$

where $L^{*}=2 L / D_{p}$. To test the validity of Eq. (21), we proceed in a similar way as done to express $h_{w p}$ from the formulation in Section 3 for monosized spheres, but taking into account that a test particle in the first layer shares more than one contact with internal particles (Eqs. (12a)-(12e) for $\Theta$ should be employed, instead of Eqs. (17a) and (17b) for $\Theta_{w}$ ), and that $h_{p L}$ is defined on the basis of the cylindrical surface at $\rho_{\mathrm{L}}$ and a driving force $\left(T^{s}-T_{c, L}^{S}\right)$ over a distance $L=\rho_{c}-\rho_{L}$. The following expression arises,

$$
h_{p L}=\frac{\pi}{4} n_{p}^{*} n_{L} \frac{\lambda_{f}}{D_{p}} 2\left(0.8 \Theta^{0}+0.2 \Theta^{0.01}\right)\left(\frac{N-1}{N-1-L^{*}}\right)
$$

where $(N-1) /\left(N-1-L^{*}\right)=\rho_{c} / \rho_{L} . \Theta^{0}$ and $\Theta^{0.01}$ are to be evaluated from Eq. (12a) with $\ell_{0} / D_{p}=0$ and 0.01 , respectively. The number of effective contacts is in this case $n_{e}=n_{L} L^{*}$, where $n_{L}$ is the average number of contacts for each particle in the first layer with inner neighbours.

It will be discussed in Section 6.1 that the total number of real contacts for a particle in the first layer can be estimated as $n_{R C, 1}=4.05$, in the range $5<N<30$. Adding near contacts with an average gap $\ell_{0}=0.01 D_{p}$, a coordination number $n_{\mathrm{co}, 1}=4.05 / 0.8=5.06$ is obtained. This value for $n_{\mathrm{co}, 1}$ includes contacts inside the first layer and those $\left(n_{L}\right)$ with inner particles. To assess the required value $n_{L}$ for Eq. (22), consider a hexagonal close-packed array of spheres that can be described as stacking close-packed layers on wall-surface. This packing structure exhibits a ratio $\left(n_{p}^{*}\right)^{1 / 2} /\left(1-\varepsilon_{c}\right)^{1 / 3}=1.188$ and a ratio $n_{L} / n_{\mathrm{co}, 1}=3 / 9$. Random beds satisfy the ratio $\left(n_{p}^{*}\right)^{1 / 2} /\left(1-\varepsilon_{c}\right)^{1 / 3}$ closely when actual values of $n_{p}^{*}$ for the first layer are considered. For example, assuming $n_{p}^{*}=0.955$ (Section 2) and $\varepsilon_{c}=0.4$, as a representative value from Eq. (5a), renders $\left(n_{p}^{*}\right)^{1 / 2} /\left(1-\varepsilon_{C}\right)^{1 / 3}=1.16$. Then we can reasonably adopt the ratio $n_{L} / n_{c o, 1}=1 / 3$. Nonetheless, when $N$ is low such a ratio should decrease due to a decreasing number of particles accommodated on inner cylindrical surfaces. Taking into account that contacts between spheres of the first layer take place at $\rho_{\mathrm{C}}$ and those between first-layer and inner spheres at $\rho_{\mathrm{L}}, n_{\mathrm{L}}$ is finally estimated from

$$
n_{\mathrm{L}}=n_{\mathrm{co}, 1} \frac{\rho_{\mathrm{L}}}{2 \rho_{\mathrm{c}}+\rho_{\mathrm{L}}}=5.06 \frac{\mathrm{N}-1-\mathrm{L}^{*}}{3(\mathrm{~N}-1)-\mathrm{L}^{*}}
$$

For example, employing Eqs. (1), (4), (5a) and $n_{p}^{*}=0.955$ to evaluate $L^{*}, n_{L}$ increases by $19 \%$ (from 1.42 to 1.69 ) as $N$ increases from 5 to $\infty$.

Eqs. (21) and (22) can now be compared. For Eq. (21), $\lambda_{\text {es, }}$ from Eq. (33) discussed later in in Section 6.2 is employed. Eqs. (1), (4b), (5a), $n_{p}^{*}=0.955$ are again used for the bed properties. Defining the relative difference $e_{p L}=\left(h_{p L(21)}-h_{p L(22)}\right) / h_{p L(22)}$ the following results varying $N$ are obtained for $\ell_{S}=0$ and $\kappa=200$ : $N=(5,7.5,15, \infty), e_{p L} \%=(-6.6,-1.2,4.2,9.2)$. These differences are acceptable, in particular noting that the effect of the region $\rho_{\mathrm{c}}<\rho<\rho_{\mathrm{L}}$ on the overall radial heat transfer rates will be modest. Additional calculations also show that the deviations $e_{p L}$ are only weakly sensitive to values of $\ell_{\mathrm{S}}$ and $\kappa$. Even taking into account a certain level of uncertainty in the values of some of the parameters, e.g. in the values of $n_{\mathrm{L}}$, Eq. (21) seems to provide a reasonable and simple estimation of $h_{p L}$ (and hence of $h_{s L}$ ). In this way, $h_{p L}$ does not introduce any additional thermal parameter, as $\lambda_{e s, c}$ needed in Eq. (21) should anyway be evaluated to describe heat transfer in the core region.

In stagnant beds it is useful to evaluate the heat transfer rate between the whole surfaces at $\rho=\rho_{\mathrm{L}}$ and that at $\rho=\rho_{\mathrm{C}}$ including the contribution of conduction in the fluid regions surrounding the particles. With a similar reasoning as that made for deriving Eq. (20) from Eq. (19), the heat transfer coefficient $h_{p L}^{0}$ between the whole surfaces is obtained from Eq. (21) as

$$
h_{p L}^{0}=\frac{\lambda_{e, c}^{0}}{\rho_{L} \ln \left(\rho_{c} / \rho_{L}\right)}=\frac{2 \lambda_{e, c}^{0}}{D_{p}\left(N-1-L^{*}\right) \ln \left(\frac{N-1}{N-1-L^{*}}\right)}
$$

where $\lambda_{e, c}^{0}$ is the effective thermal conductivity of the stagnant bed evaluated from Eq. (34), which will be discussed in Section 6.2. The final expressions to relate and estimate the coefficients $h_{s L}, h_{p L}$ and $h_{p L}^{0}$ are Eqs. (15b), (21), (24).

It is worth mentioning that up to the best of our knowledge, there is no experimental information concerning the coefficient $h_{p L}^{0}$.

\section{Evaluation of the effective thermal conductivity $\lambda_{e s, c}$}

Many studies dealing with effective thermal conductivities in packed beds can be found in literature. The review by van Antwerpen et al. (2010) covers many contributions without fluid flow until 2010, while Dixon (2012) presented a critical revision of different aspects concerning the radial heat transfer in packed bed reactors. More recent contributions about experimental results and modeling of the effective thermal conductivity are also available, as those of van Antwerpen et al. (2012), Mandal et al. (2012, 2013); Mandal and Gupta (2016), Abyzov et al. $(2013,2014)$ and Wang et al. (2016a,b). van Antwerpen et al. (2010) and Dixon (2012) concluded that the well known expression from Bauer and Schlünder (1978) is a suitable tool to predict the stagnant conductivity for the core 
region of the bed. For spheres without contact areas, $\ell^{*} \ll 1$ (i.e. excluding the case of very low pressures) and neglecting radiant heat exchange, the expression of Bauer and Schlünder (1978) becomes

$$
\begin{gathered}
\lambda_{e, \mathrm{c}}^{0} / \lambda_{f}=1-\sqrt{1-\varepsilon_{C}}+\sqrt{1-\varepsilon_{C}} \Theta_{B S} \\
\Theta_{B S}=\frac{2}{\Lambda}\left[\frac{B}{\Lambda^{2}}\left(\frac{\kappa-1}{\kappa}\right)\left(1+\ell^{*}\right) \ln \left[\frac{\kappa\left(1+\ell^{*}\right)}{B\left(1+\kappa \ell^{*}\right)}\right]\right. \\
\left.-\left(\frac{B+1}{2}\right)-\left(1+\ell^{*}\right)\left(\frac{B-1}{\Lambda}\right)\right] \\
B=1.25\left(\frac{1-\varepsilon_{C}}{\varepsilon_{C}}\right)^{10 / 9} ; \Lambda=1+\ell^{*}-B\left(\ell^{*}+1 / \kappa\right)
\end{gathered}
$$

The term $\left[\sqrt{1-\varepsilon_{C}} \Theta_{B S}\right]$ in Eq. (25a) corresponds to the contribution of particle to particle heat transfer (the dominant contribution when $\kappa \gg 1$ ), which then can be related to the parameter $\lambda_{e s, c}$ of the 2R2D model,

$$
\lambda_{e s, c} / \lambda_{f}=\sqrt{1-\varepsilon_{C}} \Theta_{B S}
$$

Formulation (25a)-(25d) is based on a cell-model with the assumption of a single contact point and flux-lines in both solid and fluid phases parallel to the direction of heat transfer. Parameter B (Eq. (25C)) represents a geometrical deformation imposed on the spheres around their contact points. This is a key parameter, as it allows simulating both, the effect of a number of contacts larger than one and the effect of the convergence of flux-lines inside the particles towards each contact point.

Another relevant feature is the factor $\left(1-\varepsilon_{c}\right)^{0.5}$ (Eq. (25d)). The origin of this factor is the complement $\left[1-\left(1-\varepsilon_{c}\right)^{0.5}\right]$, which allows expressing the effective diffusivity of a species in the fluid phase empirically as $D_{e}=\mathrm{D}\left[1-\left(1-\varepsilon_{\mathrm{c}}\right)^{0.5}\right]$, where $\mathrm{D}$ is the molecular diffusivity. The authors then assigned $\left(1-\varepsilon_{c}\right)^{0.5}$ as the fraction of area normal to the thermal flux available for particle to particle contribution. This is an ambiguous geometrical interpretation, as mass diffusion and particle-to-particle heat transfer are not equivalent (the latter involves the participation of both solid and fluid phases). Nonetheless, the combination of values assigned to parameter $B$ and the use of the factor $\left(1-\varepsilon_{c}\right)^{0.5}$ permits Formulation $(25 a)-(25 d)$ to be a good predictive tool.

We will now propose an alternative approach to evaluate $\lambda_{e s, c}$ (and also $\lambda_{e, c}^{0}$ ), based on the independent estimations of the number of contacts, the spreading/constriction of the fluxlines inside the particles, and the concentration of particles in the bed. No parameter or adjustable property to satisfy experimental values of $\lambda_{e s, c}$ (or $\lambda_{e, c}^{0}$ ) will be introduced. It is believed that such an approach should lead to a better conceptualization of the mechanism of conduction in packed beds and should also facilitate the inclusion of additional effects. For example, contact-mechanics analysis enables the evaluation of micro and macro contact areas developed between particles under the effect of compression forces. This evaluation and the subsequent thermal analysis to predict heat exchange rates between the particles requires the identification of the

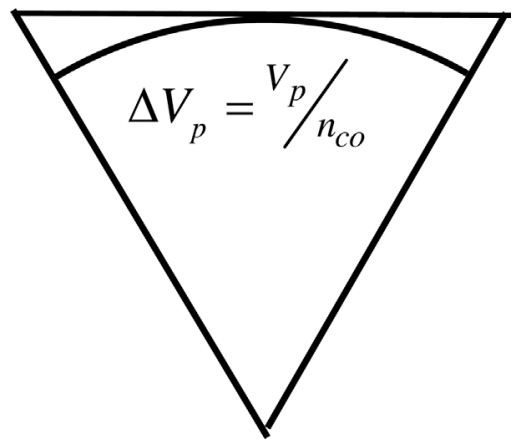

Fig. 5 - Cross-section of a cone and a sphere used to relate $n_{\mathrm{co}}$ to $\varepsilon_{\mathrm{c}}$.

number of contacts that a test particle shares in a packed bed (a comprehensive formulation is proposed by Bahrami et al. (2006)). On the other hand, Cheng and Yu (2013) have also proposed to evaluate radiation heat transfer on the basis of individual contact points.

In the following sub-section we summarize our analysis and recommendations to estimate the average number of contacts in random packed beds of monosized spheres.

\subsection{Estimation of the number of contacts}

For regular arrays of contacting spheres, the coordination number $n_{\text {co }}$ is the number of neighbors touching a sphere, while the next closest neighbors are already separated by a significantly large gap, e.g. for a simple cubic array $n_{\mathrm{co}}=6$ and the gap with the next closest neighbors is $\ell_{0}=0.41 D_{p}$. Instead of clear-cut properties, particles in random packed beds share different numbers of real contacts and the total number of neighbors continuously increases as the gap $\ell_{0}$ is allowed to rise above zero. A simplification has to be introduced to avoid that the evaluation of $\lambda_{e, c}^{0}$ inherits such a complex behavior. The approach here proposed starts by assigning a representative coordination number $n_{c o}$ to the particles in the core region, for which due account to regular arrays will be made. A fraction of this coordination number will correspond to real contacts and the remaining fraction of spheres will show some average separation gap. Both properties (fraction and average gap) will be then specified.

Consider the polyhedron defined by intersecting the planes tangent to a sphere at its $n_{\mathrm{co}}$ coordination points. Such a polyhedron can be decomposed into $n_{\text {co }}$ pyramids with the sphere centre as the common apex. Assume that a certain pyramid is representative of the whole bed in the sense that it allows writing $\varepsilon_{C}=\left(V_{P y r}-\Delta V_{p}\right) / V_{P y r}$, where $V_{P y r}$ denotes the pyramid volume and $\Delta V_{p}=V_{p} / n_{c o}$. As an approximation, we can substitute such a pyramid by a cone with the same apex and base tangent to the sphere. This cone becomes specified (see Fig. 5) by imposing that the volume intersected with the sphere satisfies $\Delta V_{p}=V_{p} / n_{c o}$. By writing $\varepsilon_{c}=\left(V_{\text {cone }}-\Delta V_{p}\right) / V_{\text {cone }}$, the proposed relation between coordination number $n_{\mathrm{co}}$ and $\varepsilon_{c}$ is obtained from elementary geometric considerations,

$$
n_{\mathrm{co}}=2(\tau-1) /(\tau-3) ; \quad \tau=\left[1+8 /\left(1-\varepsilon_{\mathrm{c}}\right)\right]^{1 / 2}
$$

It is noted that this results is the same as that employed by Abyzov et al. (2014).

Relevant values from Eq. (26) are given in Table 2, where it can be appreciated that it is able to closely approximate the values of different regular arrays. It is interesting to note that 
Table 2 - Values of coordination number $\boldsymbol{n}_{\mathrm{co}}$ from Eq. (26), and for typical regular arrays (RA).

$\begin{array}{llllllll}\varepsilon_{\text {c }} & 0.259 & 0.302 & 0.320 & 0.38 & 0.40 & 0.476 & 0.660 \\ n_{\text {co }} \text { (Eq. 26) } & 11.2 & 9.55 & 8.98 & 7.49 & 7.09 & 5.87 & 4.05 \\ n_{\text {co }} \text { (RA) } & 12 & 10 & 8 & - & - & 6 & 4 \\ \text { Regular array } & \text { Face-centered } & \text { Tetragonal- } & \text { Body- } & & & \text { Simple cubic, } & \text { Diamond } \\ & \text { cubic, FCC } & \text { spheroidal, } & \text { centered } & & & \text { SC } & \text { cubic, DC } \\ & & \text { TS } & \text { cubic, BCC } & & & \end{array}$

in the range $0.26<\varepsilon_{c}<0.5$ the values from Eq. (26) are very close to those of the even simpler expression $n_{\mathrm{co}}=1 /\left(0.35 \varepsilon_{c}\right)$.

To evaluate the number of real contacts $n_{R C}$ from the coordination number $n_{\text {co }}$ given by Eq. (26) we adopt the reasoning of Bernal and Mason (1960) in their pioneer work on contact points. They considered that in a well-compacted random packed-bed each particle should reach mechanical stability by resting on three particles and in turn supporting another three, i.e. a total of six real contacts. The authors considered a value $\varepsilon_{c}=0.38$ for a well-compacted randomly packed bed. Employing this value of $\varepsilon_{c}$, Eq. (26) gives $n_{\mathrm{co}}=7.49$ (see Table 2). Assuming the number of real contacts $n_{R C}=6$, it follows that $80 \%$ of the coordination number $n_{\mathrm{co}}$, as evaluated from Eq. (26), corresponds to real contacts $n_{R C}$. It is then proposed to adopt in general the relationship

$$
n_{R C}=0.8 n_{c o}
$$

to estimate $n_{R C}$ from a given value $n_{\text {co }}$ calculated from Eq. (26).

Eq. (27) will be appraised below in the light of experimental evidence. Next, we assess the average gap $\ell_{0}$ for the number $0.2 n_{\text {co }}$ of remaining near-contacts. To this end we employ the ratios $n_{C} / n_{R C}$ evaluated by Sahu (2009) for the total number of neighbors $n_{C}$ at increasing distances $\left(1+\ell_{0}^{*}\right) D_{p}$ between the particle centers. The author reported a set of values up to $\ell_{0}^{*}=0.08$. The following expression was found to provide the best fit to the data set

$$
n_{C} / n_{R C}=\left(1+48 \ell_{0}^{*}\right) /\left(1+28 \ell_{0}^{*}\right)
$$

According to Sahu (2009), his observations are also supported by previous studies. Employing Eq. (28) for the ratio $n_{C} / n_{R C}=1 / 0.8$ (i.e. according to Eq. (27)), we find $\ell_{0}^{*}=0.019$. Therefore, the remaining $\left(0.2 n_{\mathrm{co}}\right)$ near-contacts will present an average separation of half that value, i.e. by rounding, $\ell_{0}^{*}=0.01$.

In several studies, values of $n_{R C}$ or $n_{C}$ have been evaluated, either from direct experimental measurements or by simulating the packing of a randomly packed bed. Bernal and Mason (1960) flooded their experimental bed with a paint that after drainage left a spot around the contact points (liquid bridge technique). If the spot is annular, with the central region free from paint, it was considered that a real contact took place. On the other hand, full spots were attributed to near-contacts, assuming the paint was able to penetrate in the gaps left between neighboring particles. It is difficult to ascertain if the technique enables a clear-cut discrimination between real and near contacts, as this will depend on the ability of the paint to penetrate inside very small gaps, say when $\ell_{0}^{*}<0.01$. On these grounds, this technique might produce slight overestimations for $n_{R C}$. Employing the same experimental technique and also for a well compacted bed, Pinson et al. (1998) reported $n_{R C}=6.24$. An X-ray tomography technique was employed by Aste et al. (2005) to evaluate $n_{R C}$. Their spheres showed a size standard deviation of about $0.02 D_{p}$ (here $D_{p}$ is the average diameter) and

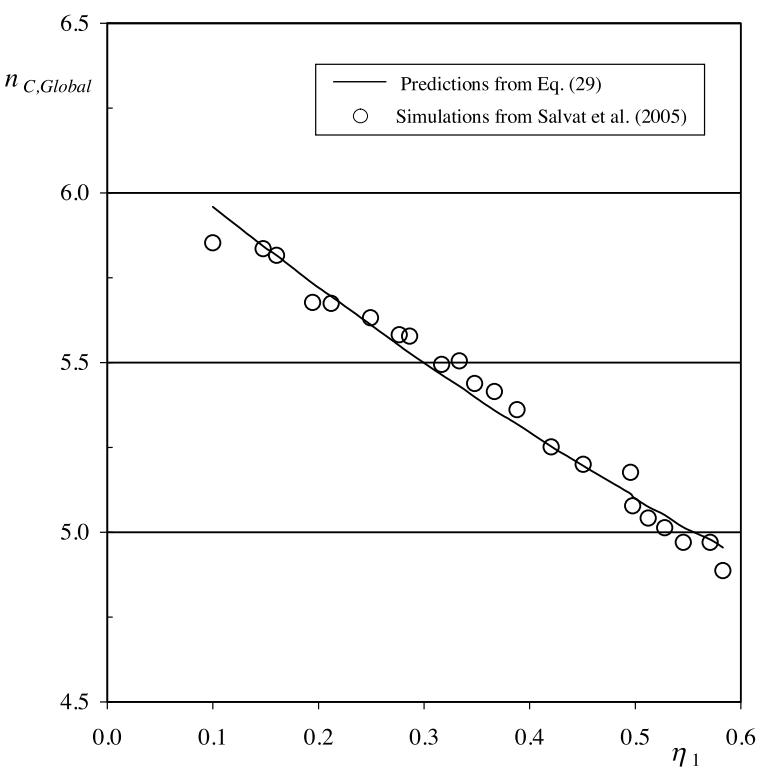

Fig. 6 - Values of $n_{C, \text { Global }}$ evaluated by Salvat et al. (2005) and predictions from Eq. (29).

hence they considered that counting particles with separation gaps of up to $\ell_{0}=0.02 D_{p}$ would represent a fair estimate of real contacts; although it was acknowledged (Aste et al., 2006) that such a criterion probably overestimates $n_{R C}$.

We also consider here three contributions that estimate $n_{R C}$ from numerical simulations of bed packings. One of them is the already cited work of Sahu (2009), who reported a value of $n_{R C}$ strictly counting contacts for $\ell_{0}^{*}=0$. Zhang et al. (2001) measured values of $n_{C}$ including gaps of up to $\ell_{0}^{*}=0.01$. The results were correlated by the authors with $\varepsilon_{c}$ according to $n_{C, 0.01}=1 /\left[0.183-659.248\left(1-\varepsilon_{C}\right)^{20.961}\right]$, valid for $0.374<\varepsilon_{C}<0.45$. From the values of $n_{C, 0.01}$, Eq. (28) can be used to compute $n_{R C}=0.865 n_{C, 0.01}$.

Finally our own results, as presented in Salvat et al. (2005), are considered. Cylindrical beds of aspect ratios $5<\mathrm{N}<30$ were simulated to evaluate the average number of contacts up to $\ell_{0}^{*}=0.005$, including those particles in the first layer around the wall. These values are denoted $n_{C, \text { Global }}$ and are plotted in Fig. 6 against the fraction of particles $\eta_{1}$ identified as belonging to the first particle layer. The fact that the number of contacts $n_{C, 1}$ for particles in the first layer should be less than in the core region, $n_{C}$, explains the trend in Fig. 6 , recalling that $\eta_{1}$ increases as $N$ decreases. It is assumed that $n_{C, 1}$ remains independent of $N$ and in the core region $n_{C}$ can be written as $n_{C}=\alpha n_{\text {co }}\left(\varepsilon_{c}\right)$, where $n_{\text {co }}\left(\varepsilon_{c}\right)$ is obtained from Eq. (26) with values $\varepsilon_{C}$ evaluated according to Eq. (5a) for a given value of $N$, and $\alpha$ is a fitting constant. Given the nearly linear dependence of $n_{\mathrm{C}, \text { Global }}$ with $\eta_{1}$, the parameters $n_{C, 1}$ and $\alpha$ have been evaluated by fitting the following expression to the experimental data:

$$
n_{C, \text { Global }}=\alpha n_{\mathrm{CO}}\left(\varepsilon_{\mathrm{C}}\right)+\left[\alpha n_{\mathrm{Co}}\left(\varepsilon_{\mathrm{C}}\right)-n_{\mathrm{C}, 1}\right] \eta_{1}
$$




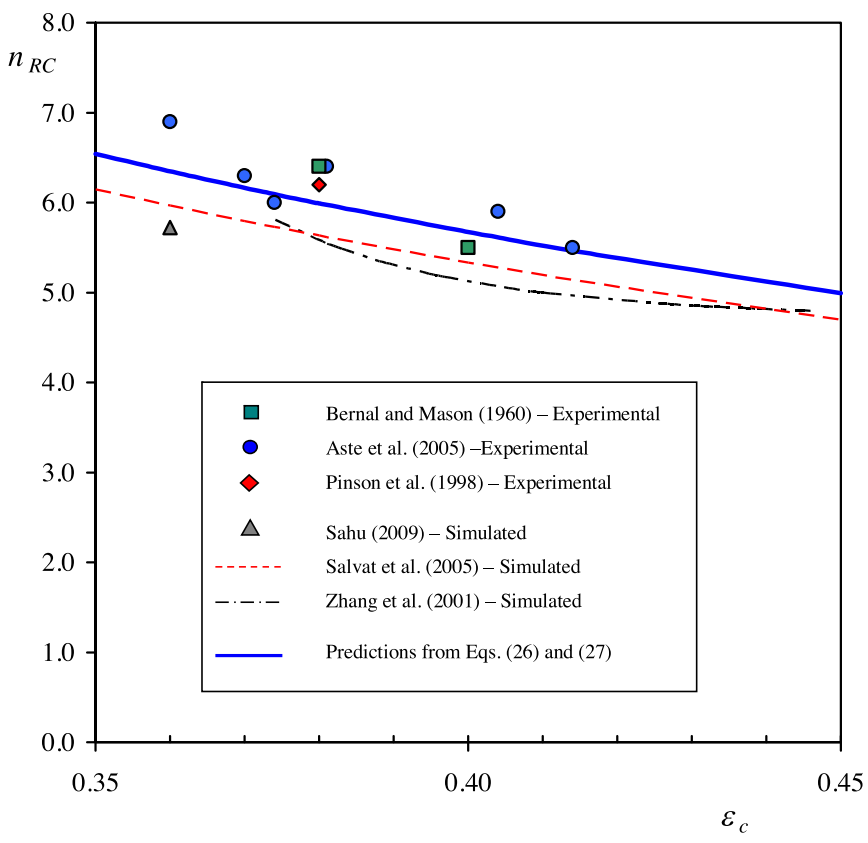

Fig. 7 - Number of real contacts $\left(n_{R C}\right)$ vs. void fraction in the core-region $\left(\varepsilon_{\mathrm{c}}\right)$.

Fitting values turned out to be $n_{C, 1}=4.4$ and $\alpha=0.82$. Eq. (29) with these parameters values is also plotted in Fig. 6 . To obtain the number of real contacts, recalling that the measurements were made at $\ell_{0}^{*}=0.005$, Eq. (28) again enables us to write for the core region $n_{R C}=0.92 n_{C}=0.92 \alpha n_{\mathrm{co}}\left(\varepsilon_{C}\right)$, and employing the fitting value of $\alpha$, the reduced data of Salvat et al. (2005) render

$$
n_{R C}=0.752 n_{c o}\left(\varepsilon_{c}\right)
$$

Results for $n_{R C}$ from the contributions discussed above are plotted in Fig. 7, along with the expression here proposed, Eq. (27) with $n_{\text {co }}$ from Eq. (26).

A reasonable agreement among the different sources can be seen in Fig. 7. Evaluations from the experimental beds are in general somewhat higher than those from the simulated beds. As discussed above, the first group of results might overestimate $n_{R C}$. Values from Eq. (27) show a good behaviour and lie approximately between both groups. The uncertainty associated with Eq. (27) in the representative range $0.37<\varepsilon_{c}<0.42$ of randomly packed beds of mono-size spheres appears to be around $\pm 5 \%$, which can be regarded as being quite acceptable for predicting effective thermal conductivities. Before closing this section, we turn back to the estimation $n_{C, 1}=4.4$ for the number of particle contacts in the first layer including gaps of up to $\ell_{0}^{*}=0.005$, according to the data of Salvat et al. (2005). The number of real contacts $n_{R C, 1}$ can be retrieved by assuming that Eq. (28) also applies to the first particle layer. In this way, $n_{R C, 1}=4.05$ is obtained. It is recalled that this value has been used in Section 5. It is also worth noting that van Antwerpen (2009) reported $n_{R C, 1}=4.35$ in a simulated annular bed with $N \approx 14$, which is in reasonable agreement with the former estimation.

\subsection{Development of new expressions for $\lambda_{e s, c}$ and $\lambda_{e, c}^{0}$}

To find an expression for $\lambda_{\text {es,c }}$, the basic result in Section 3, $\lambda_{\text {cell }} / \lambda_{f}=n_{e} \Theta$ (Eq. (10)), will be used. Two parameters should be specified: the average ( $\cos \varphi)_{a v}$ for Eq. (8) and the effective fraction of cells on a surface normal to the direction of heat transfer, $\psi$, which allows to write $\lambda_{\text {es }, c}=\psi \lambda_{\text {cell }}=\psi n_{e} \Theta$ $\lambda_{f}$. These parameters have been evaluated by Gusarov and Kovalev (2009): $(\cos \varphi)_{a v}=1 / 2$ (corresponds to an average angle $\varphi=\pi / 3$, see Fig. 2) and $\psi=\left(1-\varepsilon_{c}\right)$. Therefore,

$$
\lambda_{\text {es, },} / \lambda_{f}=\left(1-\varepsilon_{c}\right) n_{e} \Theta
$$

and

$$
n_{e}=n_{\mathrm{co}} / 4
$$

Given the relevance of parameters $(\cos \varphi)_{a v}$ and $\psi$, a derivation for them is presented in Appendix B.

In Eq. (31), $\Theta$ is evaluated from Eq. (12a), which was introduced as an approximation to the numerical results. The accuracy of the approximation, Eq. (12a), decreases up to a certain extent as $n_{e}$ increases. From Eqs. (26) and (32) $n_{e}=2$ can be taken as an upper bound (corresponds to $\varepsilon_{c}=0.357$ ) for most randomly packed beds. By scanning through values of $\kappa$, the deviation remains below $10 \%$ for $n_{e}=2$ for the most critical case $\ell^{*}=0$. For the extreme case of a FCC array $\left(\varepsilon_{c}=0.259\right.$, Table 2) $n_{e}=3$, the maximum deviation grows up to $\sim 12 \%$.

For regular arrays, it is possible to calculate exact values of $\psi$ and $(\cos \varphi)_{a v}$. Gusarov and Kovalev (2009) showed that, assuming $\Theta$ as being the same for all contacts (i.e. for large $\kappa)$, expressions (31) and (32) enable to quantify the effective conductivity exactly for the cubic arrays in Table 2 (FCC, BCC, SC, DC), as far as the exact values of $\varepsilon_{\mathrm{c}}$ and $n_{\mathrm{co}}$ (Table 2) are employed in each case. Instead, only approximate values are obtained for the tetragonal-spheroidal array, which shows thermal anisotropy.

For randomly packed beds of monosized spheres, the proposed expression for $\lambda_{e s, c}$ arises by considering $80 \%$ of real contacts and $20 \%$ of near-contacts at $\ell_{0}=0.01 D_{p}$ (Section 6.1):

$$
\lambda_{e s, c} / \lambda_{f}=\left(1-\varepsilon_{c}\right) n_{e}\left(0.8 \Theta^{0}+0.2 \Theta^{0.01}\right)
$$

where $\Theta^{0}, \Theta^{0.01}$ are evaluated from Formulation (12a)-(12e) with $\ell_{0}^{*}=0$ and $\ell_{0}^{*}=0.01$ respectively, $n_{e}$ from Eq. (32) and $n_{\mathrm{co}}$ from Eq. (26).

To obtain an expression for $\lambda_{e, c}^{0}$, it is recalled that $\Theta \rightarrow 1 / n_{e}$ if $\kappa \rightarrow 1$, and because under such condition $\lambda_{e, c}^{0}=\lambda_{f}$, a complementary term $\varepsilon_{c}$ is added to Eq. (33):

$$
\lambda_{e, c}^{0} / \lambda_{f}=\varepsilon_{c}+\left(1-\varepsilon_{c}\right) n_{e}\left(0.8 \Theta^{0}+0.2 \Theta^{0.01}\right)
$$

The term $\left(\varepsilon_{c} \lambda_{f}\right)$ in Eq. (34) accounts for the contribution of fluid conduction through the bed interstices, operating in parallel with the mechanism represented by $\lambda_{e s, c}$. The final relationships to estimate $\lambda_{e s, c}$ and $\lambda_{e, c}^{0}$ in the present contribution are given by Eqs. (33), (34).

To compare the results from Bauer and Schlünder (1978) correlation, Eq. (25a), with those from Eq. (34), the following percent difference is defined

$$
\Delta=100\left[\lambda_{e, c}^{0}\left(\text { Eq. 34) }-\lambda_{e, c}^{0} \text { (Eq. 25a) }\right] / \lambda_{e, c}^{0}\right. \text { (Eq. 25a) }
$$

Values of $\Delta$ are plotted in Fig. $8(a$ and $b)$ for $\ell_{S}^{*}=0$ and $\ell_{\mathrm{S}}^{*}=10^{-4}$ (represents air at ambient conditions and $D_{p}=2 \mathrm{~mm}$ ), respectively. The range of $\varepsilon_{c}$ in Fig. $8(\mathrm{a}$ and b) covers most possible values for packed beds, including regular arrays, while the range of $\kappa$ includes virtually 
a

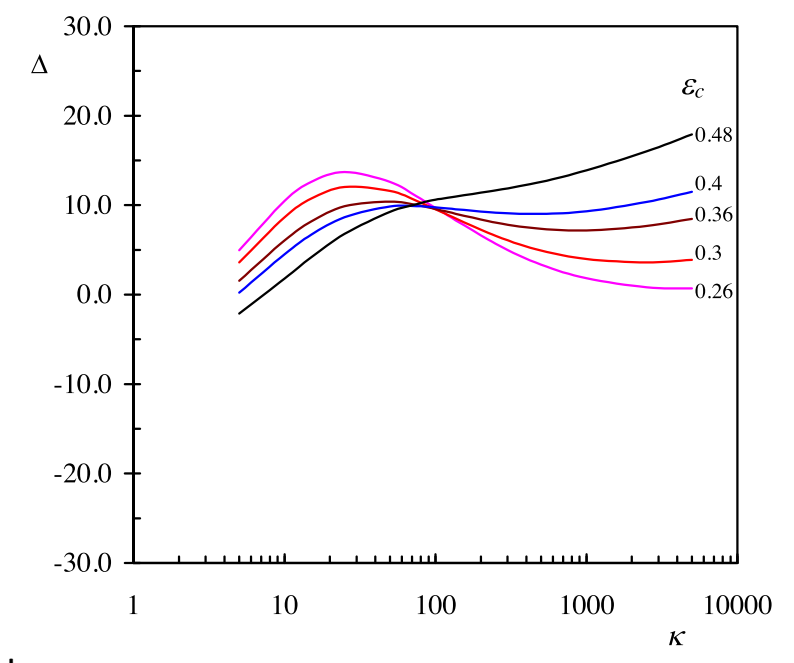

b

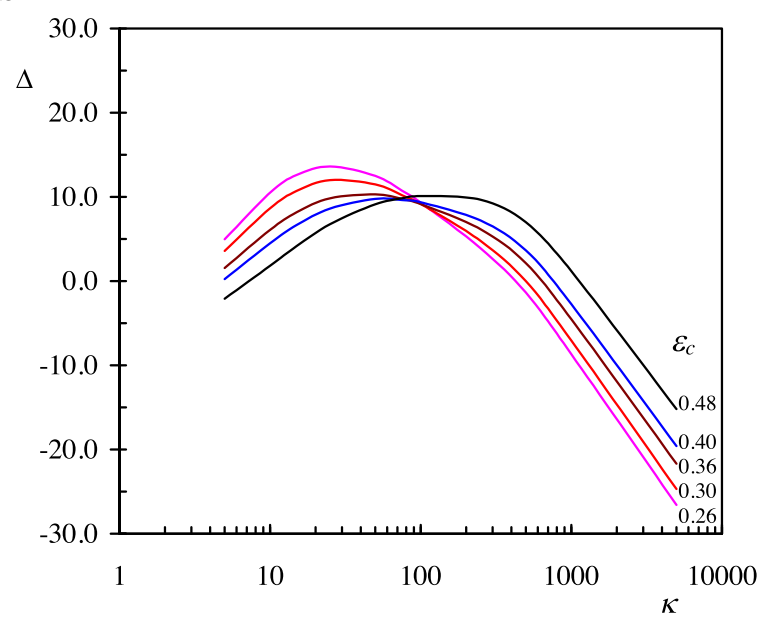

Fig. 8 - (a) Relative difference ( $\Delta$, Eq. (35)) between Eqs. (25a) and (34) for $\ell_{S}^{*}=0$. (b) Relative difference ( $\Delta$, Eq. (35)) between Eqs. (25a) and (34) for $\ell_{\mathrm{S}}^{*}=10^{-4}$.

all ceramic materials (porous or non-porous) (say, up to $\kappa=500$ ) and many metals $(500<\kappa<5000)$. For $\kappa<500$ the differences are most frequently below $10 \%$. Except at large $\varepsilon_{c}$, the differences show the same level when $\kappa>1000$ for $\ell_{S}^{*}=0$.

Larger differences, however, appear in Fig. $8 \mathrm{~b}$ at values $\kappa>1000$, when the Smoluchowski effect is considered $\left(\ell_{\mathrm{S}}^{*}=10^{-4}\right)$. This is a consequence of the fact that expression (34) is more sensitive to such an effect than the Bauer and Schlünder (1978) expression is. In turn, this effect can be explained by the fact that Bauer and Schlünder (1978) expression is based on the assumption of parallel flux lines in the solid particles.

\subsection{Comparison with experimental data}

A significant number of experimental results including those from the contributions of Bauer and Schlünder (1978), Abyzov et al. (2014) and Slavin et al. (2002) have been employed to test Eqs. (25a) and (34).

The complete set of data is reported in the Supplementary material (SM) to this contribution. Many of the data presented in the works by Bauer and Schlünder (1978) and Slavin et al. (2002) include very low pressures, virtually up to vacuum conditions. At these conditions heat conduction may

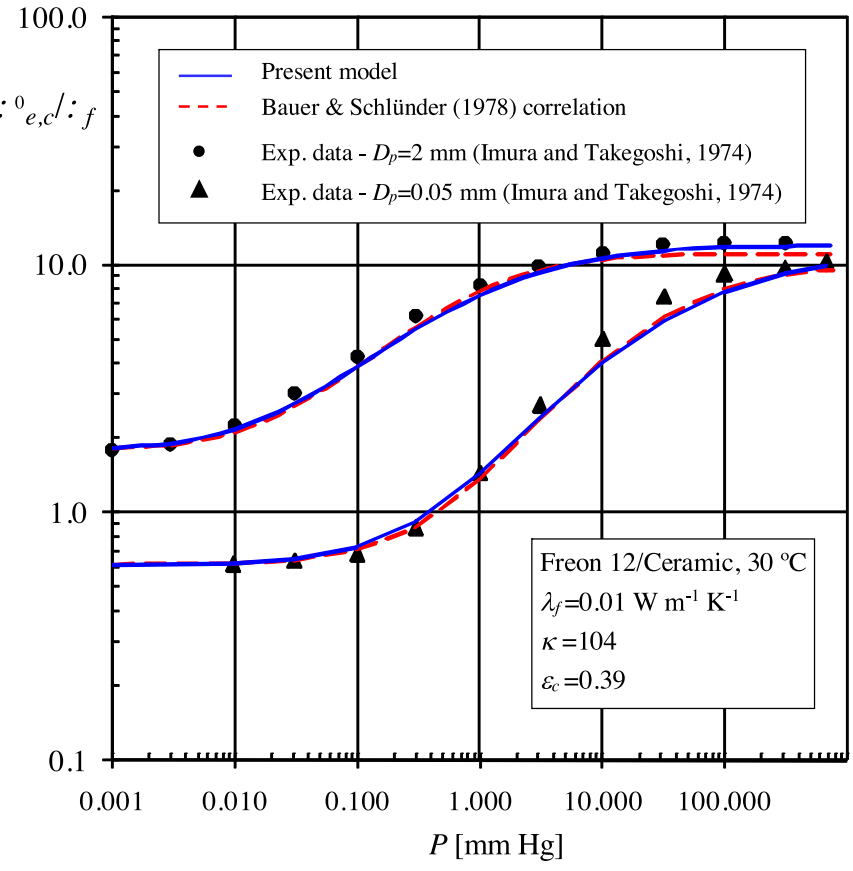

Fig. $9-\lambda_{e, c}^{0}$ vs $P$ for spherical particles.

occur in the free molecular regime, in which gas molecules travel between particle surfaces without undergoing intermolecular collisions. An expression for the effective thermal conductivity in the free molecular regime is introduced in Section S4 of the SM (see Eq. S21). The other limiting and usual case at high enough pressures is the continuum regime, in which conduction is governed by the continuum equations and gas thermal conductivity. As Eq. (34) pertains to the continuum regime, an expression for intermediate pressures matching both limiting regimes is proposed in the SM (see Eq. S23). The final equation used for predicting $\lambda_{e, c}^{0}$ (Eq. S24) also adds the heat conduction contribution due to solid-solid contact areas, which is actually the dominant contribution under vacuum conditions. This contribution was evaluated from the experimental data as the limiting value of $\lambda_{e, c}^{0}$ when full vacuum is approached. A correction to Eq. (25a) for evaluating $\lambda_{e, c}^{0}$ at low pressures has also been given by Bauer and Schlünder (1978), which is presented as Eq. (S25) of the SM.

As an example, the comparison of values of $\lambda_{e, c}^{0}$ obtained by the model proposed here and by Bauer and Schlünder (1978) correlation with the experimental data from Imura and Takegoshi (1974) (included in the article of Bauer and Schlünder (1978)) is presented in Fig. 9 for two sizes of ceramic spheres in Freon 12. It can be appreciated that both curves slightly underpredict the experimental data at intermediate pressures. This feature is found in the comparison with many of the experimental data sets presented in reference Bauer and Schlünder (1978). This shortage may be ascribed to the approximations made for the intermediate regime. Instead, for atmospheric or somewhat higher pressures the models show lower differences, without a definite bias, when compared with all of the experimental data given in references Bauer and Schlünder (1978) and Slavin et al. (2002).

It is remarked that the experimental data considered in references Bauer and Schlünder (1978) and Slavin et al. (2002) cover a wide range of conditions, involving 7 packing materials (ceramics, alumina, copper, steel, zirconium, uranium, beryllium), 7 gases (air, $\mathrm{N}_{2}, \mathrm{CH}_{4}$, Ar, Freon 12, $\mathrm{He}, \mathrm{H}_{2}$ ), and particle-size and pressures in the ranges $0.05<D_{p}<3.2 \mathrm{~mm}$, 
$0.13 \mathrm{~Pa}<\mathrm{P}<0.5 \mathrm{MPa}$. In general, Eq. (34) and its extension (Eq. S24) approximate the experimental data presented in references Bauer and Schlünder (1978) and Slavin et al. (2002) somewhat better than the Bauer and Schlünder (1978) correlation. However, the accuracy of experimental techniques and some uncertainty in properties characterizing the experimental packed beds do not allow reaching a definite conclusion about the relative merits of both models.

It is also worth to discuss briefly the effect of particle diameter on $\lambda_{e, c}^{0}$. Radiant heat transfer, free molecular conduction (at low pressures) and direct solid-solid conduction through contact areas involving asperities deformations are known to strongly increase their contributions to $\lambda_{e, c}^{0}$ as $D_{p}$ increases. Instead, at low temperatures and slight compression forces, the continuum conduction regime in the fluid determines the value of $\lambda_{e, c}^{0}$ when nearly atmospheric or higher pressures are reached. The effect of $D_{p}$ becomes mild and can be quantified by the (small) value of $\ell_{S}^{*}$ (as e.g. from Eqs. (12a), (12d)). The situation just described holds for all the experimental conditions in references Bauer and Schlünder (1978) and Slavin et al. (2002), and Fig. 9, in which the ratio between both particle sizes is 50 , provides a full representative example. In contrast, at the above described conditions, Mandal et al. (2013); Mandal and Gupta (2016) reported a strong increase of the effective thermal conductivity as $D_{p}$ increases in the range $1<D_{p}<10 \mathrm{~mm}$. Although this range includes particles bigger than those analyzed here (up to around $3 \mathrm{~mm}$ ), the effect noted in Mandal et al. (2013); Mandal and Gupta (2016) cannot be explained by the description of heat transfer mechanisms at stagnant conditions. Actually, the authors themselves recognize that large particles could have been enhanced heat transfer by natural convection (the low specific surface areas of such particles reduce friction resistances).

A different set of experimental data employed to test Eqs. (25a) and (34) involves a number of powders showing a significant size range and non-spherical shapes (Abyzov et al., 2014). Although expressions (25a) and (34) still provide reasonable estimations in this case, it appears that faceted particles with sharp edges make $\lambda_{e, c}^{0}$ increase above values for spheres. This effect can be explained by more efficient contacts between edges and facets or between facets. In this sense, Bauer and Schlünder (1978) proposed to enlarge the numerical factor defining their coefficient B (Eq. (25c)) to 1.5 for "broken particles" and to 2.5 for cylinders. A similar effect could be achieved with Eq. (34) by adjusting empirically $n_{e}$.

Eq. (34) was strictly developed on the basis of structural packing properties and numerical results describing heat conduction inside spherical particles. It is worth recalling that no thermal parameter was adjusted for the comparison with the experimental data of stagnant thermal conductivity. It is therefore important to evaluate if predicted values of $\lambda_{e, c}^{0}$ are subject to systematic deviations. To this end, 39 experimental data points for nearly spherical particles tested at atmospheric or higher pressures were selected from the experimental results presented by Bauer and Schlünder (1978), Abyzov et al. (2014) and Slavin et al. (2002), so that the conditions for which Eq. (34) was derived are maintained. The parity plot in Fig. 10 illustrates the comparison. The arithmetic mean of the individual relative errors turns out to be $e_{\mathrm{av}}=-2.5 \%$ (the corresponding average of absolute values is

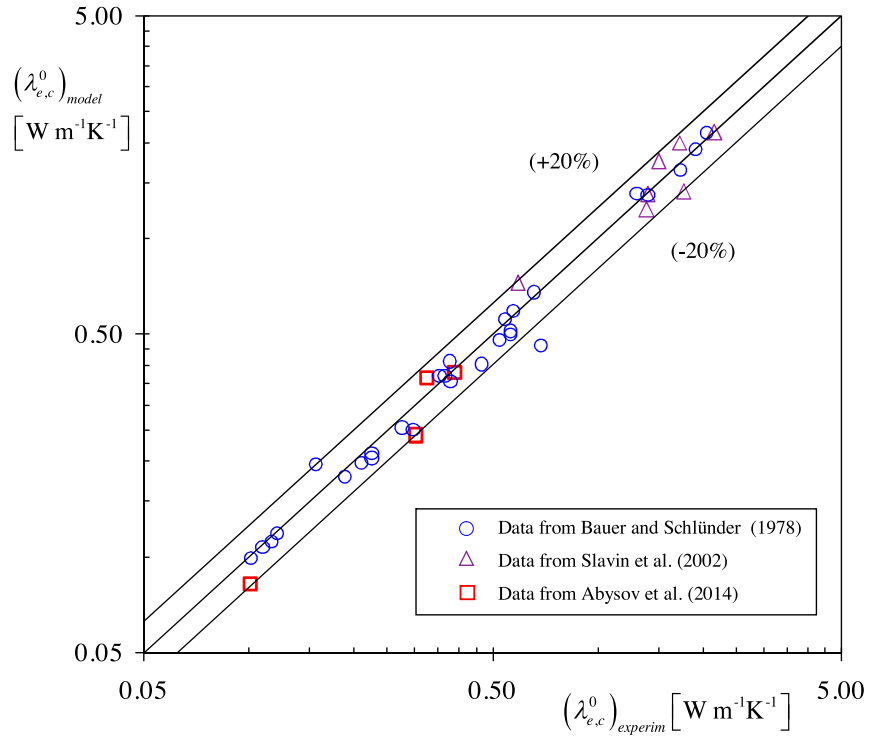

Fig. 10 - Parity plot between experimental and predicted (using Eq. (34)) values of $\lambda_{e, c}^{0}$.

$\left.|e|_{a v}=9.1 \%\right)$. The very low value of $e_{\mathrm{av}}$ indicates that the experimental data considered in this contribution do not disclose any significant systematic deviation in the behavior of Eq. (34).

\section{Conclusions}

New expressions to evaluate thermal conduction properties in fixed beds of monosized spheres have been presented and discussed in this paper. The leading motivation for undertaking this work was to evaluate the conduction parameters introduced in the 2R2D model recently proposed by Asensio et al. (2014): the effective thermal conductivity $\lambda_{e s, c}$ between particles in the inner part of the bed (core region), the heat transfer coefficient $h_{w s}$ between the particles contacting the wall (first particle layer) and the wall-surface, and the heat transfer coefficient $h_{S L}$ between such particles and the neighboring particles in the core region. Assuming local thermal equilibrium between solid and fluid phases, the parameters $\lambda_{e s, c}, h_{w s}$ and $h_{s L}$ can be associated with the corresponding stagnant properties $\lambda_{e, c}^{0}, h_{w p}^{0}$ and $h_{p L}^{0}$, which include the conduction contribution of the fluid surrounding the particles, and can be directly compared with experimental data. For simplicity, only the stagnant properties are invoked in the following paragraphs.

Expressions to evaluate the three thermal parameters have been developed on common bases:

a) The effect of the confluence of flux lines in the particles towards the contact points has been accurately approximated from numerical calculations.

b) Previous studies on the packing properties of spherical particles have enabled the evaluation of the number of particle contacts and packing densities for particles in the core region and in the first particle layer.

In this way, the formulation for $\lambda_{e, c}^{0}, h_{w p}^{0}$ and $h_{p L}^{0}$ contains only properties quantifiable beforehand, without the need for parameter fitting from the comparison with experimental data. Asensio et al. (2014) suggested that $h_{p L}^{0}$ should be related to $\lambda_{e, c}^{0}$, as expressed here by Eq. (24). Using available packing properties, $h_{p L}^{0}$ was estimated independently and the results 
showed a close agreement with the suggestion of Asensio et al. (2014). It is therefore concluded that Eq. (24) should provide a suitable estimation of $h_{p L}^{0}$.

The results from the expressions here proposed for $\lambda_{e, c}^{0}$ and $h_{w p}^{0}$ have been compared with experimental data from different sources. In either case, the analysis of the estimated values for particles of nearly spherical shapes did not reveal significant systematic deviations.

The very well known correlation of Bauer and Schlünder (1978) for $\lambda_{e, c}^{0}$ was also considered for comparison with the present expression. A parametric analysis showed that the predictions are close to each other. The Bauer and Schlünder expression was also included in the comparison with experimental data. Although somewhat better results were obtained from the formulation proposed here, it was appraised that no definite conclusion about the relative merits of both expressions can be extracted.

Experimental information employed to validate the models for $\lambda_{e, c}^{0}$ and $h_{w p}^{0}$ also involved conditions different to those assumed here for their development. Particularly for $\lambda_{e, c}^{0}$, numerous results at very low sub-atmospheric pressures were used. These conditions approach the free molecular regime (as opposed to the continuous regime at normal pressures), in which the rarefied gas molecules transport heat directly from a solid surface to another. Although the results compared with the experimental evidence can be assessed as being still satisfactory, the model tends to underpredict the values of $\lambda_{e, c}^{0}$ in the intermediate regime. Experimental values of $\lambda_{e, c}^{0}$ and $h_{w p}^{0}$ for irregular particles shapes and of $\lambda_{e, c}^{0}$ for moderately wide sizedistributions were not found to depart significantly from the predictions using the expressions and packing properties for mono-size spherical particles, but using the actual values of voidage for $\lambda_{e, c}^{0}$.

Future work is planned along two directions. First, radiation heat exchange and solid-solid conduction through finite area contacts caused by compression forces between particles are expected to be included in the formulation for spherical particles. This task may be facilitated by the existence of several studies dealing with such effects on a conceptual basis.

Finally the effect of particle shape is an important issue that deserves further examination. Cylindrical particles are of special practical significance, but a model based on similar grounds as for spheres may be exceedingly complex due to the many contact configurations between a pair of cylinders. A judicious modification of the parameters in the expressions for spheres, as e.g. the number of effective contacts, may provide a satisfactory alternative approach.

\section{Acknowledgements}

The authors wish to thank the financial support of the following Argentine institutions: CONICET (PIP \# 0018) and UNLP (PID \# 11/I177). N. J. M. and G. F. B. are Research Members of CONICET. C. D. L. holds a fellowship from CONICET.

\section{Appendix A.}

\section{Relationship between surface and volume temperature averages.}

Consider the problem of heat conduction with a source/sink term in a finite solid body of any shape, with uniform and isotropic thermal conductivity $\lambda_{p}$, volume $V_{p}$ and surface area
$S_{p}$,

$$
\lambda_{p} \nabla^{2} \mathrm{~T}+\mathrm{Q}(x, y, z)=0, \quad \operatorname{inV}_{p} ; \quad \mathrm{B}_{\mathrm{T}}(\mathrm{T})=0, \text { onS }_{p}
$$

where $B_{T}(T)=0$ stands for some boundary conditions on $S_{p}$, based on values of $T$ and/or its spatial derivatives. In Eq. (A1), the function $Q(x, y, z)$ accounts for a known distribution of specific heat generation rate inside the body. The system is decomposed in the form

$$
T=U+T_{G}
$$

where

$$
\lambda_{p} \nabla^{2} T_{G}+Q(x, y, z)=0, \quad \text { in } V_{p} ; \quad T_{G}=0, \quad \text { on } S_{p}
$$

$$
\nabla^{2} U=0, \quad \text { in } V_{p} ; \quad B_{U}(U)=0, \quad \text { on } S_{p}
$$

After solving Eq. (A3) for $T_{G}, B_{U}(U)=0$ is the proper boundary condition for $U$ that allows to satisfy $B_{T}(T)=0$ [e.g., if $B_{T}(T)=0$ is $f(x, y, z) \nabla T-T=0$ on $S_{p}, B_{U}(U)=0$ will be $f(x, y, z)\left(\nabla U+\nabla T_{G}\right)-U=0$, which accounts for $T_{G}=0$ on $\left.S_{p}\right]$.

Denoting $T_{p}$ the volume average of $T$, from Eq. (A2)

$$
\mathrm{T}_{p}=\mathrm{U}_{p}+\mathrm{T}_{G p}
$$

As we assume the problem for $T_{G}$ solved, the volume average $T_{G p}$ will be known. We express for $U_{p}$,

$$
V_{p} U_{p}=\int_{V p} U d V_{p}=-\int_{V p} U \nabla^{2} W d V_{p}
$$

where the second identity is satisfied by any field W satisfying the problem

$$
\nabla^{2} \mathrm{~W}+1=0
$$

Using the Gauss-Ostrogradsky theorem (GOT) for the last term in Eq. (A6):

$$
\mathrm{V}_{p} \mathrm{U}_{p}=\int_{\mathrm{Vp}} \nabla \mathrm{U} \cdot \nabla \mathrm{W} d \mathrm{~V}_{p}-\int_{\mathrm{Sp}} \mathrm{U} \nabla_{n} \mathrm{~W} d \mathrm{~S}_{p}
$$

where $\nabla_{n}$ is the normal component of $\nabla$ on each point of $S_{p}$. Using again the GOT for the integral on $V_{p}$ in Eq. (A8):

$$
V_{p} U_{p}=\int_{S p} W \nabla_{n} U d S_{p}-\int_{V p} W \nabla^{2} U d V_{p}-\int_{S p} U \nabla_{n} W d S_{p}
$$

The second integral is nil by virtue of Eq. (A4). Then,

$$
V_{p} U_{p}=\int_{S p} W \nabla_{n} U d S_{p}-\int_{S p} U \nabla_{n} W d S_{p}
$$

Now we use Eq. (A9) for a sphere of radius $R_{p}$. We can choose W such as its values on $S_{p}$ are nil. Then, $W=\left(R_{p}{ }^{2}-\rho^{2}\right) / 6$, with $\rho$ the radial variable in spherical coordinates. The first integral in Eq. (A9) is nil. As $\nabla_{n} W=-R_{p} / 3$, on $S_{p}$, it is obtained from Eq. 
(A9):

$$
\text { Sphere: } \quad V_{p} U_{p}=\left(R_{p} / 3\right) \int_{S p} U d S_{p}
$$

The surface averages $T^{s}$ and $U^{s}$ are defined by $T^{s}$ $S_{p}=\int_{S p} T d S_{p}, U^{s} S_{p}=\int_{S p} U d S_{p}$ and from Eq. (A2) $T^{s}=U^{s}$ (recalling that $T_{G}=0$ on $S_{p}$ ). Then, from Eq. (A10), with $V_{p} / S_{p}=R_{p} / 3$ for a sphere,

$$
\text { Sphere : } U_{p}=T^{s}
$$

From (A5):

$$
\text { Sphere : } \mathrm{T}_{p}=\mathrm{T}^{\mathrm{S}}+\mathrm{T}_{\mathrm{Gp}}
$$

Since $T_{G}=0$ if $Q=0$ in Eq. (A3), $T_{G p}=0$ in this case. Then, Eq. (A12) tells us that strictly $T_{p}=T^{s}$ is satisfied for a conduction problem in a sphere without heat source/sink, whatever the boundary condition $B_{T}(T)=0$ may be. The conclusion cannot be extended to a body shape other than spherical, although values of $T_{p}$ and $T^{s}$ will be probably similar in many cases.

\section{Appendix B.}

\section{Derivation for parameters $(\cos \varphi)_{a v}$ and $\psi$}

For a test particle in the "core region" with coordination number $n_{\mathrm{co}}$, it is assumed that the temperature gradient occurs in direction $z$ (Fig. 11) and is constant on the scale of the particle

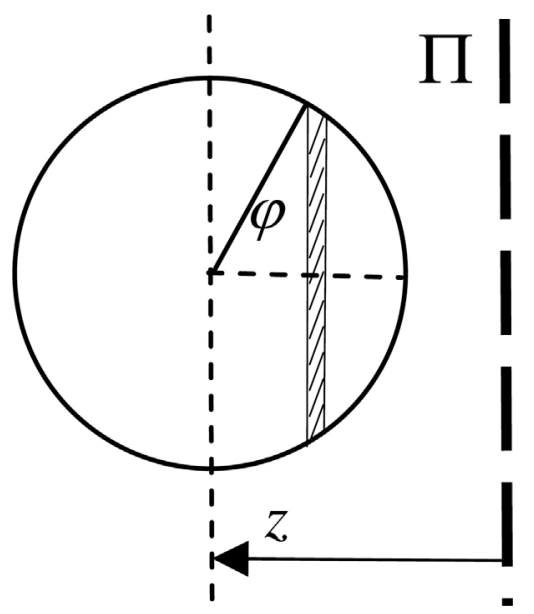

Fig. 11 - Sketch for a "test" particle in the core region.

diameter $D_{p}$.

According to Eq. (10) in the main text, the rate of heat $\Xi_{p}$ received by the right hemisphere of the test particle can be expressed as

$$
\Xi_{p}=-A_{p}^{b} n_{e} \lambda_{f} \Theta(d T / d z)
$$

To specify $n_{e}$, it is first considered that the right hemisphere will statistically present a number of contacts $n_{\mathrm{co}} / 2$. It is assumed that each of these contacts has the same probability to rest on any element of the hemisphere surface. Thus, for the surface element defined by the polar angle $\varphi$ (Fig. 11), $d A=\left(\pi D_{p}\right.$ $\sin \varphi) \frac{1}{2} D_{p} d \varphi$, the probability is $d P=d A / A_{h}$, where $A_{h}=\pi D_{p}{ }^{2} / 2$ is the area of the hemisphere. Then $d P=\sin \varphi d \varphi$.
For a contact placed at angle $\varphi$ the projection of the segment joining the particle centers in the heat transfer direction $z$ is given by $D_{p} \cos \varphi$. The dimensionless average value of the projections will be

$$
(\cos \varphi)_{a v}=\int_{0}^{\pi / 2} \cos \varphi d P=\int_{0}^{\pi / 2} \sin \varphi \cos \varphi d \varphi=1 / 2
$$

This result corresponds to an average angle $\varphi=\pi / 3$. Then, $n_{e}$ can be written as (cfr. Eq. (32))

$$
n_{e}=\left(n_{\mathrm{co}} / 2\right)(\cos \varphi)_{a v}=n_{\mathrm{co}} / 4
$$

To evaluate $\lambda_{e s, c}$, we first express the heat rate $\Xi_{\varphi}$ received by a test particle from a contact at $\varphi$,

$$
\Xi_{\varphi}=-A_{p}^{b} \lambda_{f} \Theta \cos \varphi(\mathrm{dT} / \mathrm{dz})
$$

We now intend to evaluate the rate at which heat is exchanged between particles with centers on both sides of a reference plane $\Pi$ normal to $z$ (see Fig. 11), $\Xi_{T}$. Note that the segment joining the centers of each pair of such particles should intersect plane $\Pi$. At the left of $\Pi$, the particles with centers separated at distances in the range $0<z<D_{p}$ from $\Pi$ (see Fig. 11) should be considered. Of the $n_{\mathrm{co}} / 2$ contacts on the right hemisphere of a test particle with center at $z$, only those at angles $\varphi$ less than $\varphi_{\max }$ defined as $D_{p} \cos \left(\varphi_{\max }\right)=z$ will contribute to the heat exchanged through plane $\Pi$. The heat rate received by the test particle from a contact at $\varphi \leq \varphi_{\max }$ will be given by $\Xi_{\varphi}$ (Eq. (B4)). Taking into account the probability $d P=\sin \varphi d \varphi$ for a contact at $\varphi$, the total heat rate received by the test particle through plane $\Pi$ is expressed as:

$$
\begin{gathered}
\Xi(z)=\frac{n_{\mathrm{co}}}{2}\left[-\lambda_{f} A_{p}^{b} \Theta \frac{d T}{d z}\right] \int_{0}^{\varphi_{\max }} \sin \varphi \cos \varphi d \varphi \\
=\frac{n_{\mathrm{co}}}{2}\left[-\lambda_{f} A_{p}^{b} \Theta \frac{d T}{d z}\right] \frac{1}{2}\left[1-\left(z / D_{p}\right)^{2}\right]
\end{gathered}
$$

The total heat rate $\Xi_{T}$ exchanged through the area $S$ of plane $\Pi$ is written as

$$
\Xi_{T}=n_{p}^{v} S \int_{0}^{D_{p}} \Xi(z) d z
$$

where $n_{p}^{v}$ is the number of particles per unit bed volume, $n_{p}^{v}=\left(1-\varepsilon_{c}\right) /\left(\frac{\pi}{6} D_{p}^{3}\right)$. Then,

$$
\Xi_{T}=\frac{\left(1-\varepsilon_{c}\right)}{\frac{\pi}{6} D_{p}^{3}} S\left(n_{\mathrm{co}} / 2\right)\left[-\lambda_{f} A_{p}^{b} \Theta \frac{d T}{d z}\right] \frac{1}{2} \int_{0}^{D_{p}}\left[1-\left(z / D_{p}\right)^{2}\right] d z
$$

The integral is $(2 / 3) D_{p}$. Defining $\lambda_{\text {es }, c}=-\Xi_{T} /[S(d T / d z)]$, and recalling Eq. (B3) for $n_{e}$ :

$$
\lambda_{e s, c} / \lambda_{f}=\left(1-\varepsilon_{c}\right) n_{e} \Theta
$$

By comparing Eq. (B5) with the expression $\lambda_{\text {es, }}=\psi \lambda_{\text {cell }}=\psi n_{e} \Theta$ $\lambda_{f}$, it follows that $\psi=1-\varepsilon_{c}$. 


\section{Appendix C. Supplementary Material (SM)}

Supplementary Material (SM) associated with this article can be found, in the online version, at http://dx.doi.org/10.1016/j.cherd.2017.01.012.

\section{References}

Abyzov, A.M., Goryunov, A.V., Shakhov, F.M., 2013. Effective thermal conductivity of disperse materials. I. Compliance of common models with experimental data. Int. J. Heat Mass Transfer 67, 752-767.

Abyzov, A.M., Goryunov, A.V., Shakhov, F.M., 2014. Effective thermal conductivity of disperse materials. II. Effect of external load. Int. J. Heat Mass Transfer 70, 1121-1136.

Asensio, D.A., Zambón, M.T., Mazza, G.D., Barreto, G.F., 2014. Heterogeneous two-region model for low-aspect-ratio fixed-bed catalytic reactors. Analysis of fluid-convective contributions. Ind. Eng. Chem. Res. 53, 3587-3605.

Aste, T., Saadatfar, M., Senden, T.J., 2005. Geometrical structure of disordered sphere packings. Phys. Rev. E 71, 061302.

Aste, T., Saadatfar, M., Senden, T.J., 2006. Local and global relations between the number of contacts and density in monodisperse sphere packs. J. Stat. Mech., 07010.

Bahrami, M., Yovanovich, M.M., Culham, J.R., 2006. Effective thermal conductivity of rough spherical packed beds. Int. J. Heat Mass Transfer 49, 3691-3701.

Bauer, R., Schlünder, E.U., 1978. Effective radial thermal conductivity of packing in gas flow. Part II. Thermal conductivity of the packing fraction without gas flow. Int. Chem. Eng. 18 (2), 189-204.

Bernal, J.D., Mason, J., 1960. Packing of spheres: co-ordination of randomly packed spheres. Nature 188, 910-911.

Cheng, G.J., Yu, A.B., 2013. Particle scale evaluation of the effective thermal conductivity from the structure of a packed bed: radiation heat transfer. Ind. Eng. Chem. Res. 52 (34), 12202-12211.

Dixon, A.G., 2012. Fixed bed catalytic reactor modeling. The radial heat transfer problem. Can. J. Chem. Eng. 90, 507-527.

Gusarov, A.V., Kovalev, E.P., 2009. Model of thermal conductivity in powder beds. Phys. Rev. B 80, 024202.

Imura, S., Takegoshi, E., 1974. Effect of gas pressure on effective thermal conductivity of packed beds. Trans. Jpn. Soc. Mech. Eng. 40-330, 489-497.

Kunii, D., Smith, J.M., 1960. Heat transfer characteristics of porous rocks. AIChE J. 6-1, 71-78.

Legawiec, B., Ziółkowski, D., 1994. Structure, voidage and effective thermal conductivity of solids within near-wall region of beds packed with spherical pellets in tubes. Chem. Eng. Sci. 40, 2513-2520.

Luzi, C.D., Keegan, S.D., Mariani, N.J., Barreto, G.F., 2015. Evaluation of the spreading thermal resistance for rough spheres. Int. J. Heat Mass Transfer 86, 826-831.

Mandal, D., Gupta, S., 2016. Effective thermal conductivity of unary particulate bed. Can. J. Chem. Eng. 94, 1918-1923.

Mandal, D., Sathiyamoorthy, D., Vinjamur, M., 2012. Experimental measurement of effective thermal conductivity of packed lithium-titanate pebble bed. Fusion Eng. Des. 87, 67-76.

Mandal, D., Sathiyamoorthy, D., Vinjamur, M., 2013. Void fraction and effective thermal conductivity of binary particulate bed. Fusion Eng. Des. 88, 216-225.
Marek, M., 2013. Numerical generation of a fixed-bed structure. Chem. Process Eng. 34, 347-359.

Mariani, N.J., Salvat, W.I., Martínez, O.M., Barreto, G.F., 2002. Packed bed structure. Evaluation of radial particle distribution. Can. J. Chem. Eng. 80 (2), 186-193.

Mazza, G.D., Mariani, N.J., Barreto, G.F., 1997. Evaluation of conductive heat transfer mechanisms between an immersed surface and the adjacent layer of particles in bubbling fluidized beds. Chem. Eng. Commun. 162, 93-123.

Melanson, M.M., Dixon, A.G., 1985. Solid conduction in low dt/dp fixed beds of spheres, pellets and rings. Int. J. Heat Mass Transfer 28, 383-394.

Ofuchi, K., Kunii, D., 1965. Heat-transfer characteristics of packed beds with stagnant fluids. Int. J. Heat Mass Transfer 8, 749-757.

Pinson, D., Zou, R.P., Yu, A., Zulli, P., McCarthy, M.J., 1998. Coordination number of binary mixtures of particles. J. Phys. D 31-4, 457-462.

Sahu, K.K., 2009. Gravity packing of same size spheres and investigation of wall ordering. Int. J. Chem. Reactor Eng. 7 (1), A70

Salvat, W.I., Mariani, N.J., Martínez, O.M., Barreto, G.F., 2005. On the analysis of packed bed structure at low aspect ratios. Proceeding of the ENPROMER 2005, Paper \#181, ISBN: 85-7650-043-4.

Slavin, A.J., Arcas, V., Greenhalgh, C.A., Irvine, E.R., Marshall, D.B., 2002. Theoretical model for the thermal conductivity of a packed bed of solid spheroids in the presence of a static gas, with no adjustable parameters except at low pressure and temperature. Int. J. Heat Mass Transfer 45, 4151-4161.

Tsotsas, E., Martin, H., 1987. Thermal conductivity of packed beds: a review. Chem. Eng. Process. 22, 19-37.

van Antwerpen, W., 2009. Modelling the effective thermal conductivity in the near-wall region of a packed pebble bed. In: PhD Dissertation. North-West University.

van Antwerpen, W., du Toit, C.G., Rousseau, P.G., 2010. A review of correlations to model the packing structure and effective thermal conductivity in packed beds of mono-sized spherical particles. Nucl. Eng. Des. 240, 1803-1818.

van Antwerpen, W., Rousseau, P.G., du Toit, C.G., 2012. Multi-sphere unit cell model to calculate the effective thermal conductivity in packed pebble beds of mono-sized spheres. Nucl. Eng. Des. 247, 183-201.

Wang, X., Zheng, J., Chen, H., 2016a. Application of a model to investigate the effective thermal conductivity of randomly packed fusion pebble beds. Fusion Eng. Des. 106, 40-50.

Wang, X., Zheng, J., Chen, H., 2016b. A prediction model for the effective thermal conductivity of mono-sized pebble beds. Fusion Eng. Des. 103, 136-151.

Zambón, M.T., Asensio, D.A., Barreto, G.F., Mazza, G.D., 2014. Application of computational fluid dynamics (CFD) for the evaluation of fluid convective radial heat transfer parameters in packed beds. Ind. Eng. Chem. Res. 53 (49), 19052-19061.

Zhang, Z.P., Liu, L.F., Yuan, Y.D., Yu, A.B., 2001. A simulation study of the effects of dynamic variables on the packing of spheres. Powder Tech. 116, 23-32.

Zhang, W., Thompson, K.E., Reed, A.H., Beenken, L., 2006. Relationship between packing structure and porosity in fixed beds of equilateral cylindrical particles. Chem. Eng. Sci. 61, 8060-8074. 